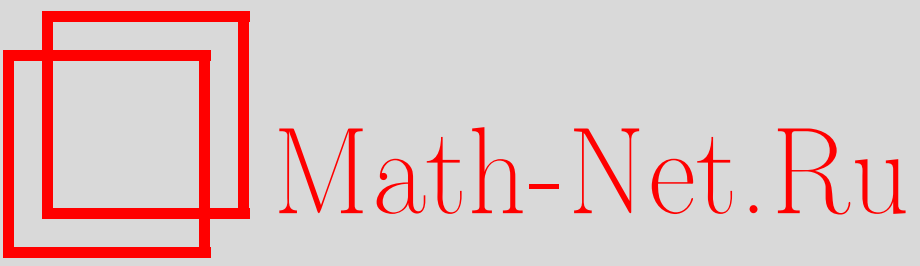

Г.-М. Гроель, Вик. С. Куликов, О симплектических накрытиях проективной плоскости, Изв. РАН. Сер. матем., 2005, том 69, выпуск 4, 19-58

DOI: https://doi.org/10.4213/im646

Использование Общероссийского математического портала Math-Net.Ru подразумевает, что вы прочитали и согласны с пользовательским соглашением

http://www . mathnet.ru/rus/agreement

Параметры загрузки:

IP: 34.229 .45 .116

26 апреля 2023 г., 12:50:47

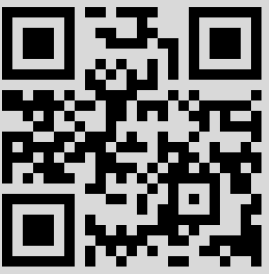




\title{
О симплектических накрытиях проективной плоскости
}

\begin{abstract}
Доказано, что разрешение особенностей произвольного конечного накрытия комплексной проективной плоскости, разветвленного вдоль кривой Гурвица $\bar{H}$ и, возможно, вдоль “бесконечно удаленной" прямой, может быть вложено как симплектическое подмногообразие в некоторое проективное алгебраическое многообразие, снабженное целочисленной симплектической кэлеровой формой (предполагается, что если $\bar{H}$ имеет отрицательные ноуды, то накрытие является неособым над ними). Для циклических накрытий это вложение может быть реализовано в некоторое рациональное комплексное трехмерное многообразие. Свойства многочленов Александера кривых Гурвица $\bar{H}$ исследованы и применены для вычисления первого числа Бетти $b_{1}\left(\bar{X}_{n}\right)$ разрешения $\bar{X}_{n}$ особенностей $n$-листного циклического накрытия $\mathbb{C P}^{2}$, разветвленного вдоль $\bar{H}$ и, возможно, вдоль “бесконечно удаленной" прямой. Доказано, что $b_{1}\left(\bar{X}_{n}\right)$ является четным числом, если $\bar{H}$ является неприводимой кривой Гурвица, и в отличие от алгебраического случая первое число Бетти может принимать любые неотрицательные значения, когда $\bar{H}$ состоит из нескольких неприводимых компонент.

Библиография: 22 наименования.
\end{abstract}

\section{Введение}

Понятие кривых Гурвица на проективной плоскости $\mathbb{C P}^{2}$ относительно линейной проекции pr: $\mathbb{C P}^{2} \backslash\left\{p_{\infty}\right\} \rightarrow \mathbb{C P}^{1}, p_{\infty}$ - центр проекции pr, было введено в [18] и является естественным обобщением понятия плоских алгебраических кривых (в [18] кривые Гурвица называются “полуалгебраическими кривыми”). Точное определение кривых Гурвица можно найти, например, в [12]. В настоящей статье мы даем другое, эквивалентное (см. лемму 1.1) определение кривых Гурвица. А именно, пусть $\mathbb{C}_{i}^{2}$ - две копии аффинной плоскости $\mathbb{C}^{2}$ с координатами $\left(u_{i}, v_{i}\right)$, $i=1,2, u_{2}=1 / u_{1}$ и $v_{2}=v_{1} / u_{1}$, которые покрьвают $\mathbb{C P}^{2} \backslash\left\{p_{\infty}\right\}$ так, что проекция $\mathrm{pr}$ задана формулами $\left(u_{i}, v_{i}\right) \rightarrow u_{i}$ в картах $\mathbb{C}_{i}^{2}$. Множество $\bar{H} \subset \mathbb{C P}^{2} \backslash\left\{p_{\infty}\right\}$, замкнутое в $\mathbb{C P}^{2}$, называется кривой Гурвица степени $m$, если для $i=1,2$ множество $\bar{H} \cap \mathbb{C}_{i}^{2}$ совпадает с множеством решений уравнения

$$
F_{i}\left(u_{i}, v_{i}\right):=v_{i}^{m}+\sum_{j=0}^{m-1} c_{j, i}\left(u_{i}\right) v_{i}^{j}=0
$$

Работа первого автора частично поддержана грантом DFG-Schwerpunkt "Globale Methoden in der komplexen Geometrie", работа второго автора - грантом РФФИ (№ 05-01-00455) и грантом DFG (436 RUS 17/84/03). 
где:

(i) $F_{i}\left(u_{i}, v_{i}\right)$ является $C^{\infty}$-гладкой комплекснозначной функцией в $\mathbb{C}^{2}$;

(ii) функция $F_{i}\left(u_{i}, v_{i}\right)$ имеет лишь конечное число критических значений, т.е. существует конечное число значений переменной $u_{i}$, скажем $u_{i, 1}, \ldots, u_{i, n_{i}}$, таких, что полиномиальное уравнение

$$
v_{i}^{m}+\sum_{j=0}^{m-1} c_{j, i}\left(u_{i, 0}\right) v_{i}^{j}=0
$$

не имеет кратных корней для $u_{i, 0} \notin\left\{u_{i, 1}, \ldots, u_{i, n_{i}}\right\}$;

(iii) если $v_{i, j}$ является кратным корнем уравнения (0.2) для $u_{i, j} \in\left\{u_{i, 1}, \ldots\right.$ $\left.\ldots, u_{i, n_{i}}\right\}$, то в окрестности точки $\left(u_{i, j}, v_{i, j}\right)$, которую мы называем критической точкой кривой $\bar{H}$, множество $\bar{H}$ совпадает с множеством решений некоторого комплексно-аналитического уравнения.

Отметим, что после перемасштабирования $\tilde{v}_{i}=\varepsilon v_{i}, 0<\varepsilon \ll 1$, кривые Гурвица становятся симплектическими поверхностями в $\mathbb{C P}^{2}$ (см. также доказательство теоремы 3.1).

Более общо, можно рассматривать так называемые топологические кривье Гурвица, которые имеют конусообразные особенности (см. определение конусообразных особенностей в [12]).

Кривая (соответственно, топологическая кривая) Гурвица $\bar{H}$ называется неприводимой, если $\bar{H} \backslash M$ связно для любого конечного множества $M \subset \bar{H}$, и мы будем говорить, что кривая Гурвища $\bar{H}$ состоит из $k$ неприводимых компонент, если

$$
k=\max \#\{\text { связные компоненты кривой } \bar{H} \backslash M\},
$$

где максимум взят по всем конечным множествам $M \subset \bar{H}$.

Пусть $H$ - аффинная кривая Гурвица, т.е. $H=\bar{H} \cap\left(\mathbb{C P}^{2} \backslash L_{\infty}\right)$, где $L_{\infty}-$ комплексная прямая, являющаяся слоем проекции pr и находящаяся в общем положении по отношению к $\bar{H}$. В этом случае фундаментальная группа $\pi_{1}=\pi_{1}\left(\mathbb{C P}^{2} \backslash\right.$ $\left.\left(\bar{H} \cup L_{\infty}\right)\right)$ не зависит от выбора прямой $L_{\infty}$ и принадлежит классу $\mathcal{C}$ так называемых $C$-групп.

По определению $C$-әруппа - это группа вместе с конечным копредставлением

$$
G_{W}=\left\langle x_{1}, \ldots, x_{m} \mid x_{i}=w_{i, j, k}^{-1} x_{j} w_{i, j, k}, w_{i, j, k} \in W\right\rangle,
$$

где $W=\left\{w_{i, j, k} \in \mathbb{F}_{m} \mid 1 \leqslant i, j \leqslant m, 1 \leqslant k \leqslant h(i, j)\right\}$ - некоторое подмножество элементов свободной группы $\mathbb{F}_{m}$ (возможно, что $w_{i_{1}, j_{1}, k_{1}}=w_{i_{2}, j_{2}, k_{2}}$ для $\left.\left(i_{1}, j_{1}, k_{1}\right) \neq\left(i_{2}, j_{2}, k_{2}\right)\right)$, порожденной свободными порождающими $x_{1}, \ldots, x_{m}$, и $h:\{1, \ldots, m\}^{2} \rightarrow \mathbb{Z}$ - некоторая функция. Такое копредставление назьвается $C$-копредставлением (буква $C$ означает, что все соотношения являются сопряжениями).

Пусть $\varphi_{W}: \mathbb{F}_{m} \rightarrow G_{W}-$ канонический эпиморфизм. Элементы $\varphi_{W}\left(x_{i}\right) \in G$, $1 \leqslant i \leqslant m$, и элементы, сопряженные им, называются $C$-порождающими әлеменmами $C$-группы $G$. Пусть $f: G_{1} \rightarrow G_{2}$ - некоторьй гомоморфизм $C$-групп. Он называется $C$-гомоморфизмом, если образы всех $C$-порождающих элементов группы $G_{1}$ при гомоморфизме $f$ являются $C$-порождающими элементами $C$-группы $G_{2}$. Мы будем рассматривать $C$-групшы с точностью до $C$-изоморфизмов. 
$C$-копредставление (0.3) называется гурвицевским $C$-копредставлением степени $m$, если для каждого $i=1, \ldots, m$ слово $w_{i, i, 1}$ совпадает с произведением $x_{1} \ldots x_{m}$, и $C$-группа $G$ называется гурвицевской $C$-группой степени $m$, если она обладает гурвищевским $C$-копредставлением степени $m$. Другими словами, $C$-группа $G$ является гурвищевской $C$-группой степени $m$, если сушествуют $C$-порождающие элементы $x_{1}, \ldots, x_{m}$, порождающие группу $G$ и такие, что произведение $x_{1} \ldots x_{m}$ принадлежит центру группы $G$. Отметим, что степень гурвицевской $C$-группы $G$ определена неканонически и зависит от ее гурвицевского $C$-копредставления. Обозначим через $\mathcal{H}$ класс всех гурвищевских $C$-групп.

Пусть $\bar{H}$ - кривая (соответственно, топологическая кривая) Гурвица степени $m$. Копредставление Зариского-ван Кампена группы $\pi_{1}=\pi_{1}\left(\mathbb{C}^{2} \backslash H\right)$, где $\mathbb{C}^{2}=$ $\mathbb{P}^{2} \backslash L_{\infty}$ и слой $L_{\infty}$ проекции pr находится в общем положении относительно кривой $\bar{H}$, определяет на $\pi_{1}$ структуру гурвищевской $C$-групшы степени $m$ (см. [11]). В работе [11] было доказано, что произвольная гурвицевская $C$-группа $G$ степени $m$ может быть реализована как фундаментальная группа $\pi_{1}\left(\mathbb{C}^{2} \backslash H\right)$ для некоторой кривой Гурвища $\bar{H}$ с особыми точками типа $w^{m}-z^{m}=0$, имеюшей степень $\operatorname{deg} \bar{H}=2^{n} m$, где $n$ зависит от гурвицевского $C$-копредставления групшы $G$. Поскольку мы рассматриваем $C$-группы с точностью до $C$-изоморфизмов, то класс $\mathcal{H}$ совпадает с классом $\left\{\pi_{1}\left(\mathbb{C}^{2} \backslash H\right)\right\}$ фундаментальных групп дополнений к аффинным кривым (соответственно, топологическим кривым) Гурвица.

Свободная группа $\mathbb{F}_{n}$ с фиксированными свободными порождаюшими элементами является $C$-группой, и для любой $C$-группы $G$ однозначно определен канонический $C$-эпиморфизм $\nu: G \rightarrow \mathbb{F}_{1}$, отображающий все $C$-порождающие элементы группы $G$ в $C$-порождающий элемент группы $\mathbb{F}_{1}$. Обозначим через $N$ его ядро. Отметим, что если все $C$-порождающие элементы $C$-группы $G$ сопряжены друг другу (такая $C$-группа называется неприводимой), то $N$ совпадает с коммутантом $^{1} G^{\prime}$.

Пусть $G-C$-группа. $C$-эпиморфизм $\nu$ индуцирует следуюшую точную последовательность групп:

$$
1 \rightarrow N / N^{\prime} \rightarrow G / N^{\prime} \stackrel{\nu_{*}}{\rightarrow} \mathbb{F}_{1} \rightarrow 1
$$

$C$-порождающий элемент группы $\mathbb{F}_{1}$ действует на $N / N^{\prime}$ сопряжением $\tilde{x}^{-1} n \tilde{x}$, где $n \in N$ и $\tilde{x}-$ один из $C$-порождаюших элементов группы $G$. Обозначим через $h$ это действие и через $h_{\mathbb{C}}$ индуцированное действие на $\left(N / N^{\prime}\right) \otimes \mathbb{C}$. Характеристический многочлен $\Delta(t)=\operatorname{det}\left(h_{\mathbb{C}}-t \mathrm{Id}\right)$ называется многочленом Александера $C$-группы $G$ (если векторное пространство $\left(N / N^{\prime}\right) \otimes \mathbb{C}$ над $\mathbb{C}$ является бесконечномерным, то по определению многочлен Александера $\Delta(t)$ тождественно равен нулю). Для (топологической) кривой Гурвица $\bar{H}$ многочлен Александера $\Delta(t)$ группы $\pi_{1}=\pi_{1}\left(\mathbb{C}^{2} \backslash H\right)$ называется многочленом Александера кривой $\bar{H}$. Отметим, что многочлен Александера $\Delta(t)$ (топологической) кривой Гурвица $\bar{H}$ не зависит от выбора общей прямой $L_{\infty}$.

Пусть $G=\left\langle x_{1}, \ldots, x_{m} \mid r_{1}, \ldots, r_{n}\right\rangle-C$-копредставление $C$-группы $G$, и пусть $\mathbb{F}_{m}$ - свободная группа, свободно порожденная $C$-порождающими элементами

\footnotetext{
${ }^{1}$ Мы используем стандартные обозначения $G^{\prime}$ для коммутанта группы $G$ и $G^{\prime \prime}$ для коммутанта группы $G^{\prime}$.
} 
$x_{1}, \ldots, x_{m}$. Обозначим через $\frac{\partial}{\partial x_{i}}$ дифференцирование Фокса [4], т. е. эндоморфизм группового кольца $\mathbb{Z}\left[\mathbb{F}_{m}\right]$ над $\mathbb{Z}$ свободной группы $\mathbb{F}_{m}$ в себя такой, что $\frac{\partial}{\partial x_{i}}: \mathbb{Z}\left[\mathbb{F}_{m}\right] \rightarrow \mathbb{Z}\left[\mathbb{F}_{m}\right]$ является $\mathbb{Z}$-линейньм отображением, удовлетворяюшим свойствам

$$
\begin{aligned}
& \frac{\partial x_{j}}{\partial x_{i}}=\delta_{i, j}, \\
& \frac{\partial u v}{\partial x_{i}}=\frac{\partial u}{\partial x_{i}}+u \frac{\partial v}{\partial x_{i}}
\end{aligned}
$$

для любых $u, v \in \mathbb{Z}\left[\mathbb{F}_{m}\right]$. Хорошо известно (доказано, например, в [19] для групп узлов и обобщено на случай произвольных $C$-групп в [9]), что многочлен Александера $\Delta(t)$ группы $G$ совпадает (с точностью до умножения на ненулевую константу и на обратимый в $\mathbb{Z}\left[t, t^{-1}\right]$ элемент $\left.\pm t^{k}\right)$ с наибольшим обшим делителем миноров порядка $m-1$ в матрице

$$
\nu_{*}\left(\frac{\partial r_{i}}{\partial x_{j}}\right) \in \operatorname{Mat}_{n \times m}\left(\mathbb{Z}\left[t, t^{-1}\right]\right),
$$

где $r_{i}, i=1, \ldots, n,-$ определяюшие соотношения для групाы $G$ и отображение $\nu_{*}: \mathbb{Z}\left[\mathbb{F}_{m}\right] \rightarrow \mathbb{Z}\left[\mathbb{F}_{1}\right] \simeq \mathbb{Z}\left[t, t^{-1}\right]$ индуцировано каноническим $C$-эпиморфизмом $\nu: \mathbb{F}_{m} \rightarrow \mathbb{F}_{1}$.

Свойства многочленов Александера плоских алгебраических кривых и их применение к вычислению первых чисел Бетти циклических накрытий проективной плоскости хорошо известны (см., например, [22], [15], [20], [6], [7], [9], [8]). Одной из целей авторов настоящей статьи является обобщение этих результатов на случай кривых Гурвица и их применение к вычислению первых чисел Бетти циклических накрытий проективной плоскости, разветвленных в кривых Гурвица.

Основные результаты этой статьи сформулированы в следуюших теоремах и следствиях.

Tеорема 0.1. Пусть $\bar{H}$ - (топологическая) кривая Гурвица степени $d$ и $\Delta(t)$ - ее многочлен Александера. Тогда:

(i) $\Delta(t) \in \mathbb{Z}[t]$

(ii) $\Delta(0)= \pm 1$;

(iii) корни многочлена $\Delta(t)$ являются корнями степени $d$ из единицы;

(iv) действие $h_{\mathbb{C}}$ на $\left(N / N^{\prime}\right) \otimes \mathbb{C}$ является полупростым.

Более того, многочлены Александера $\Delta(t)$ кривых Гурвица $\bar{H}$ степени $d$ являются делителями многочлена $(t-1)\left(t^{d}-1\right)^{d-2}$ (см. теорему 5.6), и если $\bar{H}$ состоит из $k$ неприводимых компонент, то кратность корня $t=1$ многочлена Александера $\Delta(t)$ кривой $\bar{H}$ равна $k-1$ (см. теорему 5.9).

Теорема 0.2. Если $\bar{H}$ - неприводимая (топологическая) кривая Гурвица, mo:

(i) $\Delta(t)$ является возвратным многочленом, т. е. имеет место равенство $\Delta(t)=t^{\operatorname{deg} \Delta(t)} \Delta\left(t^{-1}\right)$;

(ii) $\operatorname{deg} \Delta(t)$ является четным числом;

(iii) $\Delta(1)=1$. 
СЛЕДСТВИЕ 0.3. Пусть $\bar{H}$ - неприводимая (топологическая) кривая Гурвица степени $\operatorname{deg} \bar{H}=p^{n}$, где $p$ - простое число. Тогда:

(i) $\Delta(t) \equiv 1$;

(ii) әруппа $\pi_{1}^{\prime} / \pi_{1}^{\prime \prime}$ является конечной, әде $\pi_{1}=\pi_{1}\left(\mathbb{C}^{2} \backslash H\right)$.

Отметим также, что если $J$ - почти комплексная структура на $\mathbb{C P}^{2}$, совместимая с симплектической формой Фубини - Штуди, и $\bar{H}-J$-голоморфная кривая в $\mathbb{C P}^{2}$ степени $m$, т.е. класс $[\bar{H}]$ равен $m\left[\mathbb{C P}^{1}\right]$ в $H_{2}\left(\mathbb{C P}^{2}, \mathbb{Z}\right)$, то $\pi_{1}=\pi_{1}\left(\mathbb{C P}^{2} \backslash\right.$ $\left.\left(\bar{H} \cup L_{\infty}\right)\right)$ является гурвицевской $C$-группой степени $m$, где $L_{\infty}$ - одна из $J$-прямых, находящихся в общем положении с $\bar{H}$. Действительно, если мы выберем пучок псевдоголоморфных прямых, которому принадлежит $L_{\infty}$, то согласно теореме Зариского-ван Кампена копредставление группы $\pi_{1}$ определяется брэйд-монодромным разложением на множители кривой $\bar{H}$ относительно выбранного пучка прямых. Следовательно, $\pi_{1}$ является $C$-группой, и, аналогично случаю кривых Гурвица, легко показать (см. доказательство теоремы 6.1 в [11]), что она является гурвицевской $C$-группой степени $m$. Таким образом, многочлены Александера псевдоголоморфных кривых можно определить аналогично случаю кривых Гурвица, и они имеют те же самые свойства.

Гомоморфизм $\nu: \pi_{1} \rightarrow \mathbb{F}_{1}$, где $\pi_{1}=\pi_{1}\left(\mathbb{C}^{2} \backslash H\right)$ - фундаментальная группа дополнения к аффинной кривой Гурвища, определяет бесконечное циклическое неразветвленное накрытие $f=f_{\infty}: X_{\infty}^{\prime} \rightarrow X^{\prime}=\mathbb{C}^{2} \backslash H$. Имеем $H_{1}\left(X_{\infty}^{\prime}, \mathbb{Z}\right)=N / N^{\prime}$, и действие $h$ на $H_{1}\left(X_{\infty}^{\prime}, \mathbb{Z}\right)$ совпадает с действием порождающего элемента группы накрываюших преобразований накрытия $f_{\infty}$. Из результатов работы [13] следует, что группа $H_{1}\left(X_{\infty}^{\prime}, \mathbb{Z}\right)$ является конечно порожденной. Для произвольного $n \in \mathbb{N}$ обозначим через $\bmod _{n}: \mathbb{F}_{1} \rightarrow \mu_{n}=\mathbb{F}_{1} /\left\{h^{n}\right\}$ естественный эпиморфизм в циклическую группу $\mu_{n}$ порядка $n$. Накрытие $f_{\infty}$ может быть пропушено через циклическое накрытие $f_{n}: X_{n}^{\prime} \rightarrow \mathbb{C}^{2} \backslash H$, ассоциированное с эпиморфизмом $\bmod _{n} \circ \nu, f_{\infty}=g_{n} \circ f_{n}$. Поскольку кривая Гурвица $\bar{H}$ имеет только аналитические особенности, то накрытие $f_{n}$ может быть продолжено до гладкого отображения $\bar{f}_{n}: \bar{X}_{n} \rightarrow \mathbb{C P}^{2}$, разветвленного вдоль $\bar{H}$ и, возможно, вдоль $L_{\infty}$ (если $n$ не является делителем $\operatorname{deg} \bar{H}$, то $\bar{f}_{n}$ разветвлено вдоль $\left.L_{\infty}\right)$, где $\bar{X}_{n}$ - некоторое гладкое четырехмерное многообразие. Действие $h$ индуцирует действия $\bar{h}_{n}$ на $\bar{X}_{n}$ и $\bar{h}_{n *}$ на $H_{1}\left(\bar{X}_{n}, \mathbb{Z}\right)$.

В $\S 4$ показано (см. теорему 4.1 ), что любое такое многообразие $\bar{X}_{n}$ может быть вложено как симплектическое подмногообразие в проективное рациональное трехмерное комплексное многообразие, на котором симплектическая структура задана некоторой целочисленной кэлеровой формой.

Теорема 0.4. Пусть $\bar{X}_{n}$ - разрешение особенностей $n$-листного ииклического накрытия, разветвленного вдоль кривой Гурвица $\bar{H}$ и, возможсно, вдоль $L_{\infty}$ и ассоциированного с эпиморфизмом $\bmod _{n} \circ \nu: \pi_{1} \rightarrow \mathbb{Z} / n \mathbb{Z}$. Тогда первое число Бетти

$$
b_{1}\left(\bar{X}_{n}\right)=\operatorname{dim}_{\mathbb{C}} H_{1}\left(\bar{X}_{n}, \mathbb{C}\right)=r_{n, \neq 1},
$$

где $r_{n, \neq 1}$ - число не равных единице корней многочлена Александера $\Delta(t)$ кривой $\bar{H}$, являющихся корнями степени п из единицы.

Теоремы $0.1,0.2,0.4$ и следствие 0.3 влекут приведенные далее следствия. 
СЛЕДСТВИЕ 0.5. Пусть $\bar{X}_{n}$ - разрешение особенностей $n$-листного циклического накрытия, разветвленного вдоль кривой Гурвица $\bar{H}$ и, возможно, вдоль прямой $L_{\infty}$. Eсли $\operatorname{deg} \bar{H}$ и п взаимно просты, то $b_{1}\left(\bar{X}_{n}\right)=0$.

СлеДСТВИЕ 0.6. Пусть $\bar{X}_{n}$ - разрешение особенностей $n$-листного ичиклческого накрытия, разветвленного вдоль неприводимой кривой Гурвица $\bar{H} и$, возможнно, вдоль $L_{\infty}$. Тогда $b_{1}\left(\bar{X}_{n}\right)$ является четным числом.

Более того, мы доказываем, что для любого $k \in \mathbb{N}$ сушествует неприводимая кривая Гурвица $\bar{H}_{k}$ такая, что для некоторого $n$ (например, можно взять $n=6$; см. предложение 6.5 ) разрешение $\bar{X}_{k, n}$ особенностей $n$-листного циклического накрытия, разветвленного вдоль $\bar{H}_{k}$, имеет первое число Бетти $b_{1}\left(\bar{X}_{k, n}\right)=2 k$. Кроме того, мы показываем, что для любого $k \in \mathbb{N}$ найдется кривая Гурвища $\bar{H}_{k}$, состояшая из двух неприводимых компонент и такая, что разрешение особенностей $\bar{X}_{k, 6}$ циклического накрытия проективной плоскости, разветвленного вдоль $\bar{H}_{k}$, имеет первое число Бетти $b_{1}\left(\bar{X}_{k, 6}\right)=k$. Напомним, что $b_{1}\left(\bar{X}_{n}\right)$ всегда является четным числом, если $\bar{H}$ является плоской алгебраической кривой, поэтому эти $\bar{H}_{k}$ не могут быть алгебраическими, если $k$ нечетно. Другие примеры кривых Гурвица, не изотопных алгебраическим кривым, можно найти в работе ${ }^{2}$ [18].

СЛЕДСТВИЕ 0.7. Пусть $\bar{X}_{n}-$ разрешение особенностей ииклического накрытия проективной плоскости, разветвленного вдоль кривой Гурвица $\bar{H}$, состоящей из $k$ неприводимых компонент, $u$, возможно, вдоль $L_{\infty}$. Если $\operatorname{deg} \bar{H}$ является делителем $n$, то $b_{1}\left(\bar{X}_{n}\right)=\operatorname{deg} \Delta(t)-k+1$.

СлеДСТВИЕ 0.8. Пусть $\bar{X}_{n}$ - разрешение особенностей ииклического накрытия проективной плоскости произвольной степени $n$, разветвленного вдоль неприводимой кривой Гурвица $\bar{H}$ и, возможно, вдоль $L_{\infty}$. Eсли степень $\operatorname{deg} \bar{H}=p^{k}$, где $p$ - простое число, то $b_{1}\left(\bar{X}_{n}\right)=0$.

СлеДСТВИЕ 0.9. Пусть $\bar{X}_{p^{k}}$ - разрешение особенностей ииклического накрытия проективной плоскости степени $p^{k}$, разветвленного вдоль неприводимой кривой Гурвица $\bar{H}$ и, возможно, вдоль $L_{\infty}$, где $p$ - простое число. Тогда $b_{1}\left(\bar{X}_{p^{k}}\right)=0$.

Отметим, что для любого $k \in \mathbb{N}$ мы доказываем сушествование кривой Гурвица $\bar{H}_{k}$, состоящей из $k+1$ неприводимых компонент, такой, что разрешение $\bar{X}_{k, 2}$ особенностей двулистного циклического накрытия плоскости имеет $b_{1}\left(\bar{X}_{k, 2}\right)=k$ (см. предложение 6.6). В частности, в нашем примере кривая Гурвица $\bar{H}_{1}$ имеет $\operatorname{deg} \bar{H}_{1}=2^{10}$, число особых точек кривой $\bar{H}_{1}$ равно $2^{16}$ и все ее особые точки являются особыми точками типа $w^{4}-z^{4}=0$.

Недавно Д. Ору и Л. Катзарков (см. [1], [2]) доказали следующую теорему. Пусть $(X, \omega)$ - компактное симплектическое четырехмерное многообразие с сим-

\footnotetext{
${ }^{2}$ Мойшезон в [18] доказал существование бесконечной последовательности неприводимых каспидальных кривых Гурвица $\bar{H}_{i}$ степени 54 , имеющих ровно 378 каспов и 756 ноудов. Каждая пара кривых в этой последовательности имеет различные типы брэйд-монодромии. В частности, эти кривые попарно не изотопны и почти все из них не изотопны алгебраическим каспидальным кривым.
} 
плектической формой $\omega$, класс которой $[\omega] \in H^{2}(X, \mathbb{Z})$. Зафиксируем $\omega$-совместимую почти комплексную структуру $J$ и соответствующую риманову метрику $g$. Пусть $L$ - линейное расслоение на $\bar{X}$, первый класс Черна которого совпадает с [ $\omega]$. Тогда для $k \gg 0$ линейное расслоение $L^{\otimes k}$ имеет достаточно много почти голоморфных сечений, так что можно выбрать три из них, дающих почти голоморфное общее накрытие $f_{k}: \bar{X} \rightarrow \mathbb{C P}^{2}$ степени $N_{k}=k^{2} \omega^{2}$, разветвленное вдоль каспидальной кривой Гурвица $\bar{H}_{k}$ (возможно, с отрицательными ноудами).

Любое такое накрытие $f_{k}: \bar{X} \rightarrow \mathbb{C P}^{2}$ степени $N_{k}$, разветвленное вдоль каспидальной кривой Гурвица $\bar{H}$, определяет монодромию $\mu$, т.е. эпиморфизм $\mu$ : $\pi_{1}\left(\mathbb{C}^{2} \backslash H\right) \rightarrow \Sigma_{N_{k}}$ в симметрическую группу $\Sigma_{N_{k}}$, удовлетворяющий некоторым дополнительным свойствам общности накрытия. С другой стороны, любой гомоморфизм $\mu: \pi_{1}\left(\mathbb{C}^{2} \backslash H\right) \rightarrow \Sigma_{N}$ такой, что $\mu\left(\pi_{1}\right)$ действует транзитивно на множестве, состоящем из $N$ элементов, определяет неразветвленное накрытие $f: X \rightarrow \mathbb{C}^{2} \backslash H$ степени $N$. Накрытие $f$ может быть продолжено до накрытия $\tilde{f}: \widetilde{X} \rightarrow \mathbb{C P}^{2}$, разветвленного вдоль кривой Гурвица $\bar{H}$ и, возможно, вдоль $L_{\infty}$. В настоящей статье мы доказываем (см. следствие 3.2 ), что если $\widetilde{X}$ имеет произвольные аналитические особенности (и если $\bar{H}$ имеет отрицательные ноуды, то мы предполагаем, что накрываюшее пространство неособо над ними), то разрешение $\bar{X}$ особенностей пространства $\widetilde{X}$ может быть снабжено симплектической структурой.

Доказательства теорем 0.1, 0.2 и следствия 0.3 приведены в $\S 5$, а $\S 6$ посвящен доказательству теоремы 0.4 .

Второй автор выражает свою благодарность Университету г. Кайзерслаутерн, во время пребывания в котором была сделана данная работа.

\section{$\S 1$. Представление кривых Гурвица в виде нулей сечений линейных расслоений}

Вначале докажем следующую лемму.

ЛЕмма 1.1. Определения кривых Гурвица на проективной плоскости $\mathbb{C P}^{2}$, даннье во введении настоящей статьи и в работе [12], являются эквивалентными.

ДокАЗАТЕЛЬСтво. Напомним определение кривых Гурвища, данное в [12]. Пусть $F_{1}$ - относительно минимальная линейчатая поверхность, $\operatorname{pr}: F_{1} \rightarrow \mathbb{C P}^{1}-$ морфизм, задаюший линейчатую структуру, $R$ - слой морфизма pr и $E_{1}-$ исключительное сечение, $E_{1}^{2}=-1$. Отождествим pr: $F_{1} \rightarrow \mathbb{C P}^{1}$ с линейной проекцией $\mathrm{pr}: \mathbb{C P}^{2} \rightarrow \mathbb{C P}^{1}$ из точки $p \in \mathbb{C P}^{2}$ ( $p$ является образом кривой $E_{1}$ при его стягивании в точку).

Согласно определению, данному в [12], образ $\bar{H}=f(S) \subset F_{1}$ гладкого отображения $f: S \rightarrow F_{1} \backslash E_{1}$ ориентированной замкнутой вещественной поверхности $S$ называется кривой Гурвица (относительно проекции pr) степени $m$, если найдется конечное подмножество $Z \subset \bar{H}$ такое, что:

(i) $f$ является вложением поверхности $S \backslash f^{-1}(Z)$ и для каждой точки $s \notin Z$ поверхность $\bar{H}$ и слой $R_{\operatorname{pr}(s)}$ проекции pr пересекаются в $s$ трансверсально с положительным индексом пересечения; 
(ii) для каждой точки $s \in Z$ найдется окрестность $U \subset F_{1}$ этой точки такая, что $\bar{H} \cap U$ является комплексно-аналитической кривой и комплексная ориентация на $\bar{H} \cap U \backslash\{s\}$ совпадает с ориентацией, индуцированной с $S$ отображением $f$;

(iii) ограничение проекции pr на $\bar{H}$ является конечным отображением степени $m$.

Чтобы показать, что определение, данное во введении, влечет определение из [12], осушествим несколько моноидальных преобразований с центрами в особых точках кривой $\bar{H}$ (и в особых точках собственных прообразов кривой $\bar{H}$ ), разрешающих все особые точки кривой $\bar{H}$. Обозначим через $\sigma: \widetilde{\mathbb{C P}}^{2} \rightarrow \mathbb{C P}^{2}$ композицию этих моноидальных преобразований и через $S$ собственньй прообраз вешественной поверхности $\bar{H}$. Тогда $S$ является гладкой вешественной поверхностью и $f=\sigma_{\mid S}$ является гладким отображением. Чтобы определить ориентацию на $S$, выберем ориентацию в каждой некритической точке $p$ проекции $\operatorname{pr}_{\mid \bar{H}}$ так, чтобы локальный индекс пересечения поверхности $\bar{H}$ и слоя $R$, проходящего чеpeз $p$, был равен +1 в точке $p$. Очевидно, эти ориентации совместимы для всех некритических точек кривой $\bar{H}$. Поскольку вблизи критических точек эта ориентация совпадает с ориентацией, заданной комплексно-аналитической структурой (напомним, что $\bar{H}$ является комплексно-аналитической кривой вблизи критических точек проекции $\left.\operatorname{pr}_{\mid \bar{H}}\right)$, эта ориентация может быть продолжена в прообразы этих критических точек.

Чтобы показать, что определение из [12] влечет определение, данное во введении, выберем слой $R_{\infty}$ проекции pr и положим $\mathbb{C}^{2}=F_{1} \backslash\left(R_{\infty} \cup E_{1}\right)$. Пусть $(u, v)$ - такие координаты в $\mathbb{C}^{2}$, что ограничение проекции $\mathrm{pr}$ задается формулой $(u, v) \rightarrow u$. Пусть $\left(u, v_{1}(u)\right), \ldots,\left(u, v_{m}(u)\right)$ - координаты точек пересечения кривой $\bar{H}$ и слоя $R$ проекции $\mathrm{pr}$ над некритическим значением $u$. Рассмотрим

$$
F(u, v)=\prod_{i=1}^{m}\left(v-v_{i}(u)\right)
$$

Очевидно, функция $F(u, v)$, определенная всюду вне слоев над критическими значениями, является гладкой и может быть продолжена до функции на всем $\mathbb{C}^{2}$, удовлетворяющей всем условиям из данного во введении определения.

Пусть кривая Гурвица $\bar{H}_{0}$ степени $m$ задана уравнениями $(0.1)$. Гладкая изотопия $h_{t}: \mathbb{C P}^{2} \times[0,1] \rightarrow \mathbb{C P}^{2} \times[0,1]$ называется $H$-изотопией, если для каждого $t \in[0,1]$ образ $\bar{H}_{t}=h_{t}\left(\bar{H}_{0}\right)$ является кривой Гурвица, заданной уравнениями

$$
v_{i}^{m}+\sum_{j=0}^{m-1} c_{j, i}\left(u_{i}, t\right) v_{i}^{j}=0, \quad i=1,2,
$$

$c_{j, i}\left(u_{i}, 0\right)=c_{j, i}\left(u_{i}\right)$ для всех $i, j$. (Отметим, что в определении $H$-изотопии, данном в [12], предполагается дополнительно, что число критических значений кривых $\bar{H}_{t}$ не зависит от $t$.) Легко видеть, что если $\bar{H}_{0}$ и $\bar{H}_{1} H$-изотопны, а прямая $L_{\infty}$ находится в общем положении по отношению к обеим кривым Гурвища $\bar{H}_{0}$ и $\bar{H}_{1}$, то $\mathbb{C}^{2} \backslash H_{0}$ и $\mathbb{C}^{2} \backslash H_{1}$ диффеоморфны. 
Обозначим через $p_{\infty}=\mathbb{C P}^{2} \backslash\left(\mathbb{C}_{1}^{2} \cup \mathbb{C}_{2}^{2}\right)$ центр проекции pr. Далее мы будем предполагать, что слой проекции рr над точкой $u_{2}=0$ является общим относительно кривой $\bar{H}_{0}$. Обозначим его через $L_{\infty}$. Очевидно, существует гладкая $H$-изотопия $h_{t}$, тождественная вне малой окрестности $U$ прямой $L_{\infty}$, такая, что функция $F_{2}\left(u_{2}, v_{2}, 1\right)$, задаюшая кривую $\bar{H}_{1}=h_{1}\left(\bar{H}_{0}\right)$ в $\mathbb{C}_{2}^{2}$, совпадает с функцией $v_{2}^{m}-1$ во всех точках $\left(u_{2}, v_{2}\right)$ при $\left|u_{2}\right|<\varepsilon$ для некоторого $\varepsilon>0$. Далее мы будем предполагать, что

(*) $L_{\infty}$ задается уравнением $u_{2}=0$ и кривая $\bar{H}$ - уравнением $v_{2}^{m}-1=0$ в некоторой окрестности прямой $L_{\infty}$.

Пусть $u_{1, j}-$ критическое значение кривой Гурвища $\bar{H}_{0}$ степени $m$, заданной в $\mathbb{C}_{1}^{2}$ уравнением $F_{1}\left(u_{1}, v_{1}\right)=0$, т. е. число различных корней уравнения

$$
F_{1}\left(u_{1, j}, v_{1}, 0\right)=0
$$

строго меньше $m$, и пусть $v_{1, j_{0}}$ - корень уравнения (1.2) кратности один. Очевидно, существует гладкая $H$-изотопия $h_{t}$, тождественная вне малой окрестности $U=$ $\left\{\left|u_{1}-u_{1, j}\right|<\varepsilon\right\}$, такая, что функция $F_{1}\left(u_{1}, v_{1}, 1\right)$, определяющая $\bar{H}_{1}=h_{1}\left(\bar{H}_{0}\right)$ в $\mathbb{C}_{1}^{2}$, такова, что $v_{1}=v_{1, j_{0}}$ является корнем уравнения $F_{1}\left(u_{1}, v_{1}, 1\right)=0$ для всех $u_{1}$, удовлетворяюших неравенству $\left|u_{1}-u_{1, j}\right|<\varepsilon_{1}$ при некотором положительном $\varepsilon_{1}<\varepsilon$. Поэтому далее мы можем (и будем) предполагать, что если $\left(u_{1, j}, v_{1, j}\right)$ является критической точкой кривой $\bar{H}$, то

$\left.{ }^{* *}\right)$ существует такое $\varepsilon>0$, ито функиия $F_{1}\left(u_{1}, v_{1}\right)$, определяющая кривую $\bar{H}$, является аналитической в точках $\left(u_{1}, v_{1}\right)$ при $\left|u_{1}-u_{1, j}\right|<\varepsilon u\left|v_{1}-v_{1, j}\right|<\varepsilon$.

Рассмотрим линейное расслоение $p: \mathcal{L}(k) \rightarrow \mathbb{C P}^{2}$, ассоциированное с пучком $\mathcal{O}_{\mathbb{C P}^{2}}(k)$. Напомним его определение. Проективная плоскость $\mathbb{C P}^{2}$ с однородными координатами $\left(z_{0}: z_{1}: z_{2}\right)$ покрьвается тремя картами $\mathbb{C}_{i}^{2}, i=1,2,3$, изоморфными $\mathbb{C}^{2}$, с координатами $\left(u_{i}, v_{i}\right), \quad u_{1}=z_{1} / z_{0}, \quad v_{1}=z_{2} / z_{0}, u_{2}=z_{0} / z_{1}$, $v_{2}=z_{2} / z_{1}, u_{3}=z_{0} / z_{2}, \quad v_{3}=z_{1} / z_{2}$. Расслоение $\mathcal{L}(k)$ также покрыто тремя картами $W_{i}=\mathbb{C}_{i}^{2} \times \mathbb{C}_{i}^{1}$ с третьей координатой $w_{i}, w_{1}=w_{2} / u_{2}^{k}, w_{1}=w_{3} / u_{3}^{k}$, $w_{2}=w_{3} / v_{3}^{k}$, и ограничение $p_{\mid W_{i}}$ совпадает с проекцией на первую координату.

Лемма 1.2. Функиии $w_{i}=F_{i}\left(u_{i}, v_{i}\right), \quad i=1,2$, определяющие кривую $\bar{H}$, задают гладкое сечение s расслоения $\mathcal{L}(m)$ над $\mathbb{C P}^{2} \backslash\left\{p_{\infty}\right\}$.

ДокАЗАТЕльство. В пересечении карт $\mathbb{C}_{1}^{2} \cap \mathbb{C}_{2}^{2}$ имеем

$$
\begin{aligned}
F_{1}\left(u_{1}, v_{1}\right) & =v_{1}^{m}+\sum_{j=0}^{m-1} c_{j, 1}\left(u_{1}\right) v_{1}^{j}=\left(\frac{v_{2}}{u_{2}}\right)^{m}+\sum_{j=0}^{m-1} c_{j, 1}\left(\frac{1}{u_{2}}\right)\left(\frac{v_{2}}{u_{2}}\right)^{j} \\
& =\left(\frac{1}{u_{2}}\right)^{m}\left(v_{2}^{m}+\sum_{j=0}^{m-1} c_{j, 1}\left(\frac{1}{u_{2}}\right) u_{2}^{m-j} v_{2}^{j}\right)
\end{aligned}
$$

Функции

$$
F_{2}\left(u_{2}, v_{2}\right)=v_{2}^{m}+\sum_{j=0}^{m-1} c_{j, 2}\left(u_{2}\right) v_{2}^{j}
$$


и

$$
v_{2}^{m}+\sum_{j=0}^{m-1} c_{j, 1}\left(\frac{1}{u_{2}}\right) u_{2}^{m-j} v_{2}^{j}
$$

совпадают друг с другом, так как они являются гладкими и для почти всех (за исключением конечного числа) значений $u_{2,0}$ переменной $u_{2}$ многочлены

$$
v_{2}^{m}+\sum_{j=0}^{m-1} c_{j, 2}\left(u_{2,0}\right) v_{2}^{j}
$$

и

$$
v_{2}^{m}+\sum_{j=0}^{m-1} c_{j, 1}\left(\frac{1}{u_{2,0}}\right) u_{2,0}^{m-j} v_{2}^{j}
$$

имеют одно и то же множество корней.

Лемма 1.3. Пусть $f_{0}: S^{3} \rightarrow \mathbb{C}^{*}=\mathbb{C} \backslash\{0\}-$ гладкая функиия, заданная на $S^{3}=\left\{(u, v) \in \mathbb{C}^{2} \mid u \bar{u}+v \bar{v}=\varepsilon^{2}\right\}, \quad 0<\varepsilon \ll 1$, такая, что $f_{0}$ совпадает c функичей $v^{m}-1$ в некоторой окрестности $U \subset S^{3}$ окружности $u=0$. Тогда существует гладкая функиия $F: S^{3} \times[0,1] \rightarrow \mathbb{C}^{*}$ такая, ито:

(i) $F(u, v, 0)=f_{0}(u, v)$;

(ii) $F(u, v, t)=v^{m}-1$ для $(u, v) \in U$ u всех $t \in[0,1]$;

(iii) $F(u, v, 1)=v^{m}-1$.

ДокАЗАТЕЛЬСтво. Поскольку сфера $S^{3}$ является односвязной, то существует подъем $\tilde{f}_{0}: S^{3} \rightarrow \widetilde{\mathbb{C}}^{*}$ функции $f_{0}$ такой, что $f_{0}=e \circ \tilde{f}_{0}$, где $\widetilde{\mathbb{C}}^{*}-$ комплексная плоскость $\mathbb{C}$ с комплексной координатой $x$, а $e: \widetilde{\mathbb{C}}^{*} \rightarrow \mathbb{C}^{*}-$ универсальное накрытие, заданное функцией $y=e^{x}$. Не ограничивая обшности, мы можем предполагать, что $\tilde{f}_{0}(0, \varepsilon)=\ln \left(1-\varepsilon^{m}\right)+\pi i$. Обозначим через $f_{1}: S^{3} \rightarrow \mathbb{C}^{*}$ функцию $v^{m}-1$ и через $\tilde{f}_{1}: S^{3} \rightarrow \widetilde{\mathbb{C}}^{*}-$ ее подъем такой, что $\tilde{f}_{1}(0, \varepsilon)=\ln \left(1-\varepsilon^{m}\right)+\pi i$. Имеем $\tilde{f}_{0 \mid U} \equiv \tilde{f}_{1 \mid U}$

Рассмотрим функцию $\widetilde{F}: S^{3} \times[0,1] \rightarrow \mathbb{C}$, заданную формулой

$$
x=t \tilde{f}_{1}(u, v)+(1-t) \tilde{f}_{0}(u, v) .
$$

Очевидно, функция $F=e$ ○ $\widetilde{F}$ обладает всеми требуемыми свойствами.

Лемма 1.4. Существуют вещественное число $\varepsilon_{1}, \quad 0<\varepsilon_{1} \ll 1$, и гладжое сечение $\bar{s}_{m}$ расслоения $\mathcal{L}(m)$ над $\mathbb{C P}^{2}$ такие, что:

(i) $\bar{H} \subset \mathbb{C P}^{2} \backslash B\left(\varepsilon_{1}\right)$, где $B\left(\varepsilon_{1}\right)=\left\{u_{3} \bar{u}_{3}+v_{3} \bar{v}_{3} \leqslant \varepsilon_{1}^{2}\right\}-$ некоторыи ииар в $\mathbb{C P}^{2}$ с иентром в $p_{\infty}$

(ii) над $\mathbb{C P}^{2} \backslash B\left(\varepsilon_{1}\right)$ сечение $\bar{s}_{m}$ совпадает с сечением $s$ из леммы 1.2 ;

(iii) сечение $\bar{s}_{m}$ является комплексно-аналитическим в некоторой окрестности прямой $L_{\infty}$.

ДокАЗАТЕльство. Согласно определению кривых Гурвица сушествует шар $B\left(\varepsilon_{1}\right)=\left\{u_{3} \bar{u}_{3}+v_{3} \bar{v}_{3} \leqslant \varepsilon_{1}^{2}\right\}$ при некотором положительном $\varepsilon_{1}$ такой, что $\bar{H} \subset \mathbb{C P}^{2} \backslash$ $B\left(\varepsilon_{1}\right)$. 
Линейное расслоение $\mathcal{L}_{m}$ является тривиальным над $B\left(\varepsilon_{1}\right)$. Следовательно, ограничение сечения $s$ из леммы 1.2 на $\partial B\left(\varepsilon_{1}\right)=S^{3}$ задается функцией $f_{0}$ : $S^{3} \rightarrow \mathbb{C}^{*}$. Обозначим через $F: S^{3} \times[0,1] \rightarrow \mathbb{C}^{*}$ функцию, существование которой доказано в лемме 1.3 (в обозначениях леммы 1.3 положим $u=u_{3}, v=v_{3}$ и $\varepsilon=\varepsilon_{1}$ ). Выберем $\varepsilon_{2}<\varepsilon_{1}$ и гладкую монотонную функцию $r:\left[\varepsilon_{2}, \varepsilon_{1}\right] \rightarrow[0,1]$ такую, что $r\left(\varepsilon_{1}\right)=0$ и $r\left(\varepsilon_{2}\right)=1$. Обозначим через $h: B\left(\varepsilon_{1}, \varepsilon_{2}\right)=B\left(\varepsilon_{1}\right) \backslash B\left(\varepsilon_{2}\right) \rightarrow \partial B\left(\varepsilon_{1}\right)$ отображение, заданное равенством

$$
h\left(u_{3}, v_{3}\right)=\left(\frac{\varepsilon_{1} u_{3}}{\sqrt{u_{3} \bar{u}_{3}+v_{3} \bar{v}_{3}}}, \frac{\varepsilon_{1} v_{3}}{\sqrt{u_{3} \bar{u}_{3}+v_{3} \bar{v}_{3}}}\right),
$$

положим $\widetilde{F}\left(u_{3}, v_{3}, t\right)=h^{*}(F)$ и

$$
\bar{F}\left(u_{3}, v_{3}\right)=\left(\frac{\sqrt{u_{3} \bar{u}_{3}+v_{3} \bar{v}_{3}}}{\varepsilon_{1}}\right)^{m}\left(\widetilde{F}\left(u_{3}, v_{3}, r\left(\sqrt{u_{3} \bar{u}_{3}+v_{3} \bar{v}_{3}}\right)\right)+1\right)-1 .
$$

Тогда сечение $\tilde{s}$, заданное следуюшим образом:

$$
\tilde{s}(p)= \begin{cases}s(p) & \text { для } p \in \mathbb{C P}^{2} \backslash B\left(\varepsilon_{1}\right), \\ \bar{F}\left(u_{3}, v_{3}\right) & \text { для } p \in B\left(\varepsilon_{1}\right) \backslash B\left(\varepsilon_{2}\right), \\ v_{3}^{m}-1 & \text { для } p \in B\left(\varepsilon_{2}\right),\end{cases}
$$

удовлетворяет всем условиям леммы 1.4, за исключением, возможно, того, что оно не является гладким, а только непрерывным, в точках из $B=\left(\partial B\left(\varepsilon_{1}\right) \cup \partial B\left(\varepsilon_{2}\right)\right) \backslash U$, где $U$ - некоторая окрестность прямой $L_{\infty}$. Применяя стандартные теоремы математического анализа, легко показать, что существует сечение $\bar{s}_{m}$, достаточно близкое к $\tilde{s}$, совпадаюшее с $\tilde{s}$ вне достаточно малой окрестности $V$ шара $B$ такой, что $\bar{V} \cap\left(\bar{H} \cup L_{\infty}\right)=\varnothing$, где $\bar{V}$ - замыкание множества $V$.

\section{$\S 2$. Симплектические многообразия с аналитическими особенностями}

Пусть $Y$ - неособое проективное комплексное многообразие комплексной размерности $\operatorname{dim}_{\mathbb{C}} Y=n$ и $\omega-$ кэлерова форма на $Y,[\omega] \in H^{2}(Y, \mathbb{Z})$. Рассмотрим симплектическое многообразие $(Y, \omega), \operatorname{dim}_{\mathbb{R}} Y=2 n$. Замкнутое подмногообразие $X$ в $Y$ называется симплектическим многообразием с аналитическими особенностями, если сушествуют открытые подмножества $U_{0} \subset U \subset Y$ такие, что замыкание $\bar{U}_{0}$ в $Y$ содержится в $U, X \cap U$ является комплексно-аналитическимподмножеством в $U$ и $X \backslash \bar{U}_{0}$ является гладким симплектическим подмногообразием. Обозначим через $\operatorname{Sing} X$ множество точек из $X$, в которых $X$ не является гладким. Тогда Sing $X$ является проективным алгебраическим подмногообразием в $Y$.

ЛЕмма 2.1. Пусть $X$ - симплектическое многообразие с аналитическими особенностями в комплексном проективном многообразии $Y$ с кәлеровой формой $\omega$. Пусть $Z \subset \operatorname{Sing} X$ - неособое проективное подмногообразие в $Y$, $\sigma: \bar{Y} \rightarrow Y$ - моноидальное преобразование многообразия $Y$ с иентром в $Z$ и $\bar{X}$ - собственный прообраз многообразия $X$. Тогда существует кәлерова форма $\bar{\omega}$ на $\bar{Y}$ такая, что $\bar{X}$ является симплектическим многообразием с аналитическими особенностями в $(\bar{Y}, \bar{\omega})$. 
ДокАЗАТЕльСтво. Многообразие $\bar{Y}$ является проективным алгебраическим. Рассмотрим некоторое вложение $i: \bar{Y} \hookrightarrow \mathbb{C P}^{N}$ в какое-нибудь проективное пространство и обозначим через $\varphi=i \circ \sigma^{-1}$ рациональное отображение из $Y$ в $\mathbb{C P}^{N}$. Пусть $\Gamma \subset Y \times \mathbb{C P}^{N}$ - замыкание графика отображения $\varphi$ и $p_{i}, \quad i=1,2$, проекции из $Y \times \mathbb{C P}^{N}$ на множители. Морфизмы $i$ и $\sigma$ определяют морфизм $\sigma \times i: \bar{Y} \rightarrow \Gamma \subset Y \times \mathbb{C P}^{N}$. Поскольку композиция $p_{2} \circ(\sigma \times i): \bar{Y} \rightarrow \bar{Y}$ является изоморфизмом, то $p_{2 \mid \Gamma}: \Gamma \rightarrow \bar{Y}$ также является изоморфизмом. Более того, если мы отождествим $\bar{Y}$ с $\Gamma$ с помошью $p_{2 \mid \Gamma}$, то $p_{1 \mid \Gamma}$ будет совпадать с $\sigma$.

Обозначим через $\Omega=\Omega_{N}$,

$$
\Omega_{N}=\frac{i}{\left(\sum_{j=0}^{N} \bar{z}_{j} z_{j}\right)^{2}} \sum_{k=0}^{N} \sum_{j \neq k}\left(\bar{z}_{j} z_{j} d z_{k} \wedge d \bar{z}_{k}-\bar{z}_{j} z_{k} d z_{j} \wedge d \bar{z}_{k}\right),
$$

симплектическую форму Фубини-Штуди на $\mathbb{C P}^{N}$. Она является целочисленной кэлеровой формой. Рассмотрим ограничение формы $\omega_{\varepsilon}=p_{1}^{*}(\omega)+\varepsilon p_{2}^{*}(\Omega)$ на $\Gamma$. Она является кэлеровой формой на Г при каждом $\varepsilon>0$.

Выберем открытые окрестности $V_{0} \subset V \subset Y$ множества $\operatorname{Sing} X$ такие, что $V \cap X$ является аналитическим подмногообразием и замыкание $\bar{V}_{0}$ множества $V_{0}$ в $Y$ содержится в $V$. Обозначим $X_{0}=X \backslash V_{0}$. Это множество является компактом, и $\sigma_{\mid X_{0}}^{-1}: X_{0} \rightarrow \bar{X}_{0}=\sigma^{-1}\left(X_{0}\right)$ является изоморфизмом. Следовательно, $\bar{X}_{0}$ является компактом.

Очевидно, ограничение формы $\omega_{\varepsilon}$ на $\Gamma \cap p_{1}^{-1}(V)$ является симплектической формой в каждой точке из $\Gamma \cap p_{1}^{-1}(V)$ для всех $\varepsilon>0$, так как $\Gamma \cap p_{1}^{-1}(V)$ является аналитическим подмножеством в $p_{1}^{-1}(V)$. Поскольку ограничение формы $\omega$ на $X_{0}$ является симплектической формой в каждой точке из $X_{0}$ и $\bar{X}_{0}$ является компактом, то мы можем выбрать достаточно маленькое $\varepsilon$ такое, что ограничение формы $\omega_{\varepsilon}=p_{1}^{*}(\omega)+\varepsilon p_{2}^{*}(\Omega)$ на $\bar{X}_{0}=\Gamma \cap p_{1}^{-1}\left(X_{0}\right)$ является симплектической формой в каждой точке из $\bar{X}_{0}$. Если мы возьмем рациональное $\varepsilon=\frac{m}{n}$, то $n \omega_{\varepsilon}$ будет целочисленной формой.

\section{§3. Симплектичность накрытий проективной плоскости, разветвленных вдоль кривых Гурвица}

В этом параграфе мы используем обозначения и предположения из $\S 1$.

Пусть $\bar{H}$ - кривая Гурвица, возможно, с отрицательныцми ноудами, т. е. в некоторой окрестности $U$ каждой критической точки $p$ либо $\bar{H}$ локально задается аналитическим уравнением, либо $\bar{H} \cap U$ состоит из двух гладких ветвей, пересекаюшихся трансверсально в точке $p$ с индексом пересечения, равным -1 , и каждая ветвь кривой $\bar{H} \cap U$ пересекает слой $\operatorname{pr}^{-1}(\operatorname{pr}(p))$ в точке $p$ трансверсально с индексом пересечения, равным +1 .

Зафиксируем точку $p \in \mathbb{C P}^{2} \backslash\left(\bar{H} \cup L_{\infty}\right)$. Рассмотрим фундаментальную группу $\pi_{1}=\pi_{1}\left(\mathbb{C}^{2} \backslash H, p\right)$ дополнения к аффинной кривой Гурвица $H=\left(\mathbb{C P}^{2} \backslash L_{\infty}\right) \cap \bar{H}$. Выберем точку $x \in \bar{H} \backslash \operatorname{Sing} \bar{H}$ и рассмотрим комплексную прямую $L \subset \mathbb{C}^{2}$, пересекающую $H$ трансверсально в точке $x$. Пусть $\gamma \subset L$ - окружность малого радиуса с центром в $x$. Выбор ориентации на $\mathbb{C}^{2}$ определяет ориентацию на $\gamma$. Пусть $\Gamma$ - замкнутый путь, состоящий из пути $l$ в $\mathbb{C}^{2} \backslash H$, соединяющего точку $p$ с точкой $q \in \gamma$, 
петли $\gamma$ (обходимой в положительном направлении) с началом и концом в точке $q$ и обратного пути в точку $p$ вдоль $l$ в противоположном направлении. Такая петля $\Gamma$ (и соответствующий элемент в $\pi_{1}$ ) называется геометрическим порождающим элементом (с центром в $x$ ) фундаментальной группы $\pi_{1}=\pi_{1}\left(\mathbb{C}^{2} \backslash H, p\right)$. Хорошо известно, что $\pi_{1}$ порождается геометрическими порождающими элементами.

Для каждой критической точки $s_{i}$ кривой $H$ выберем окрестность $U_{i} \subset \mathbb{C}^{2}$ такую, что $H \cap U_{i}$ либо задается (в локальных координатах на $U_{i}$ ) аналитическим уравнением, либо, если $s_{i}$ - отрицательный ноуд, состоит из двух гладких ветвей, пересекающихся трансверсально в точке $p$. Заметим, что если $s_{i}$ - отрищательный ноуд, то группа $\pi_{1}\left(U_{i} \backslash H, p_{i}\right)$ изоморфна группе $\mathbb{Z} \oplus \mathbb{Z}$ и порождается двумя коммутирующими геометрическими порождающими элементами.

Выберем гладкие пути $\gamma_{i}$, лежащие в $\mathbb{C}^{2} \backslash H$ и соединяющие точки $p_{i}$ с точкой $p$. Этот выбор определяет гомоморфизмы $\psi_{i}: \pi_{1}\left(U_{i} \backslash H, p_{i}\right) \rightarrow \pi_{1}$. Назовем $\psi_{i}\left(\pi_{1}\left(U_{i} \backslash H, p_{i}\right)\right)=G_{i}$ локальной фундаментальной әруппой критической точки $s_{i}$. Локальные фундаментальные групшы определены однозначно с точностью до сопряжения в $\pi_{1}$.

Рассмотрим такой гомоморфизм $\mu: \pi_{1} \rightarrow \Sigma_{N}$ из фундаментальной группы $\pi_{1}=$ $\pi_{1}\left(\mathbb{C}^{2} \backslash H\right)$ дополнения к аффинной кривой Гурвища $H=\left(\mathbb{C P}^{2} \backslash L_{\infty}\right) \cap \bar{H}$ в симметрическую группу $\Sigma_{N}$, что его образ $\operatorname{Im} \mu$ действует транзитивно на множестве, состоящем из $N$ элементов.

Пусть $s_{i}$ - отрицательный ноуд кривой $H$. Как было упомянуто выше, локальная фундаментальная группа $G_{i}$ порождается двумя коммутирующими геометрическими порождаюшими элементами, скажем $\Gamma_{i, 1}$ и $\Gamma_{i, 2}$. Обозначим

$$
N_{i, j}=\left\{1 \leqslant n \leqslant N \mid \mu\left(\Gamma_{i, j}\right)(n) \neq n\right\} .
$$

Скажем, что гомоморфизм $\mu$ является хорошим в отрииательном ноуде $s_{i}$, если $N_{i, 1} \cap N_{i, 2}=\varnothing$. Гомоморфизм $\mu$ называется монодромией степени $N$, если он является хорошим во всех отрицательных ноудах.

Гомоморфизм $\mu$ определяет неразветвленное накрытие $f=f_{\mu}: Y \rightarrow \mathbb{C}^{2} \backslash H$ степени $N$. Это накрытие может быть продолжено до конечного разветвленного накрытия $\tilde{f}: \widetilde{Y} \rightarrow \mathbb{C P}^{2}$ с ветвлением вдоль $\bar{H}$ и, возможно, вдоль $L_{\infty}$.

Для того чтобы описать это продолжение, рассмотрим одну из геометрических порождающих $\Gamma$ с центром в точке $x \in H \backslash$ Crit $H$, где Crit $H$ - множество критических точек кривой $H$. Образ $\mu(\Gamma)$ в $\Sigma_{N}$ является произведением циклических перестановок $\sigma_{1}, \ldots, \sigma_{n_{x}}$ (возможно, что порядки некоторых из них равны единище). Каждая из этих перестановок $\sigma_{l}$ циклически переставляет элементы в множестве $\left\{n_{1, l}, \ldots, n_{r_{l}, l}\right\}, 1 \leqslant n_{j, l} \leqslant N$, где $r_{l}$ - порядок перестановки. Тогда число прообразов $\tilde{f}^{-1}(x)$ равно $n_{x}$ и каждая точка $y$ из $\tilde{f}^{-1}(x)$ соответствует некоторой циклической перестановке $\sigma_{l}$. Вблизи точки $y_{l}$, соответствующей циклической перестановке $\sigma_{l}$, накрытие $\tilde{f}$ является циклическим накрытием степени $r_{l}$, разветвленным вдоль $H$ и локально изоморфным подмногообразию в $\mathbb{C}^{3}$, заданному уравнением $w^{r_{l}}=v-v_{j}(u)$, где $v-v_{j}(u)=0$ - локальное уравнение кривой $\bar{H}$ в точке $x$ (см. (1.1)). Эти локальные изоморфизмы определяют на $\tilde{Y}$ структуру гладкого многообразия в каждой точке $y$, лежащей над точкой $x \in H \backslash$ Crit $H$. 
Пусть $s_{i} \in$ Crit $H$ является отрицательным ноудом. Как было упомянуто выше, локальная фундаментальная группа $G_{i}$ порождается двумя геометрическими порождающими элементами $\Gamma_{i, 1}$ и $\Gamma_{i, 2}$. Образы $\mu\left(\Gamma_{i, j}\right)$ в $\Sigma_{N}$ являются произведениями циклических перестановок $\sigma_{1, i, j}, \ldots, \sigma_{k_{i, j}, i, j}$. Пусть

$$
\sigma_{l, i, j}=\left(n_{1, l, i, j}, \ldots, n_{r_{l, i, j}, l, i, j}\right)
$$

- перестановка порядка $r_{l, i, j}$. Положим $N_{i, 1,2}=\left\{1 \leqslant n \leqslant N \mid \mu\left(\Gamma_{i, 1}\right)(n)=n\right.$ и $\left.\mu\left(\Gamma_{i, 2}\right)(n)=n\right\}$. Поскольку $\mu$ является монодромией, множество $\tilde{f}^{-1}\left(s_{i}\right)$ находится во взаимно однозначном соответствии с объединением всех циклических перестановок $\sigma_{l, i, j}, j=1,2$, имеющих порядки больше единицы, и множества $N_{i, 1,2}$. Более того, если $y \in \tilde{f}^{-1}\left(s_{i}\right)$ соответствует элементу из $N_{i, 1,2}$, то $\tilde{f}$ является изоморфизмом некоторой окрестности $V$ точки $y$ и ее образа $\tilde{f}(V)$, и если $y \in \tilde{f}^{-1}\left(s_{i}\right)$ соответствует циклической перестановке $\sigma_{l, i, j}$ порядка больше единищы, то ограничение накрывающего отображения $\tilde{f}$ на некоторую окрестность точки $y$ является циклическим накрытием некоторой окрестности точки $s_{i}$ степени $r_{l, i, j}$, разветвленным в $j$-й ветви отрицательного ноуда. Это накрытие локально изоморфно многообразию в $\mathbb{C}^{3}$, заданному уравнением $w^{r_{l, i, j}}=v-v_{j}(u)$, где $v-v_{j}(u)=0$ локальное уравнение $j$-й ветви кривой $\bar{H}$ в точке $s_{i}$. Эти локальные изоморфизмы определяют на $\tilde{Y}$ структуру гладкого многообразия в каждой точке $y \in \tilde{f}^{-1}\left(s_{i}\right)$.

Пусть $x=s_{i} \in \operatorname{Crit}_{\text {analytic }} H$, т. е. $s_{i}$ - критическая точка кривой $H$, не являющаяся отрищательным ноудом. Тогда над небольшой окрестностью $U$ точки $s_{i}$, в которой кривая $H$ задается аналитическим уравнением, прообраз $\tilde{f}^{-1}(U)$ является несвязным объединением $n_{s_{i}}$ открытых окрестностей, находящихся во взаимно однозначном соответствии с элементами из множества орбит действия группы $\mu\left(G_{i}\right)$ на множестве, состоящем из $N$ элементов. Согласно теореме Грауерта-Реммерта-Штейна (доказательство см. в [21]) над окрестностью $U$ точки $s_{i}$ многообразие $\widetilde{Y}$ может быть снабжено структурой двумерного комплексно-аналитического многообразия.

По предположению в некоторой окрестности $U$ прямой $L_{\infty}\left(\right.$ где $U=\mathbb{C P}^{2} \backslash B(R)$ и $B(R) \subset \mathbb{C}_{1}^{2}$ - шар большого радиуса $R$ ) кривая $\bar{H}$ совпадает с алгебраической кривой $\bar{C} \subset \mathbb{C P}^{2}$ степени $m$, заданной в $U$ уравнением $v_{2}^{m}-1=0$. Если мы выберем базисную точку $p$ лежащей в $U$, мы можем рассмотреть копредставления Зариского-ван Кампена группы $\pi_{1}$ и фундаментальной группы $\tilde{\pi}_{1}=\pi_{1}\left(\mathbb{C P}^{2} \backslash\left(\bar{C} \cup L_{\infty}\right), p\right)$, имеющие одни и те же множества порождающих элементов, и легко видеть, что эти представления определяют эпиморфизм $e: \widetilde{\pi}_{1} \rightarrow \pi_{1}$. Композищия $\mu \circ e$ определяет разветвленное накрытие $\tilde{g}: \widetilde{Z} \rightarrow \mathbb{C P}^{2}$ с ветвлением вдоль $\bar{C}$ и, возможно, вдоль $L_{\infty}$. Легко видеть, что накрытия $\tilde{f}$ и $\tilde{g}$ изоморфны над $U$. Следовательно, многообразие $\tilde{f}^{-1}(U)$ может быть отождествлено с $\tilde{g}^{-1}(U)$ с помощью изоморфизма $h: \tilde{f}^{-1}(U) \rightarrow \tilde{g}^{-1}(U)$. Таким образом, мы можем рассматривать $\tilde{f}^{-1}(U)$ как комплексно-аналитическое многообразие.

Пусть $i: \widetilde{Z} \hookrightarrow \mathbb{C P}^{m_{\infty}}$ - вложение такое, что $\tilde{g}$ задается проекцией

$$
\left(z_{0}^{\prime}: z_{1}^{\prime}: \ldots: z_{m_{\infty}}^{\prime}\right) \rightarrow\left(z_{0}^{\prime}: z_{1}^{\prime}: z_{2}^{\prime}\right)
$$


Положим

$$
\begin{array}{ll}
z_{j}=h^{*}\left(z_{j}^{\prime}\right), & j=3, \ldots, m_{\infty}, \\
w_{j, \infty}^{\prime}=\frac{z_{j}}{z_{0}}, & j=3, \ldots, m_{\infty} .
\end{array}
$$

Теорема 3.1. Пусть $\bar{H}$ - кривая Гурвица с отрицательным.ми ноудами, $\mu: \pi_{1}\left(\mathbb{C}^{2} \backslash H\right) \rightarrow \Sigma_{N}-$ монодромия и $\tilde{f}: Y \rightarrow \mathbb{C P}^{2}-$ накритие, ассоциированное с монодромией $\mu$. Тогда $\widetilde{Y}$ может бить вложено в некоторое проективное пространство $\mathbb{C P}^{M}$ как симплектическое подмногообразие с аналитическими особенностями.

ДоКАЗАТЕЛЬСтво. Ниже для каждой точки $p \in \mathbb{C}_{1}^{2}$ обозначим через $V_{p} \subset U_{p}$ небольшие шары $V_{p}=\left\{\left|u_{1}-u_{1}(p)\right|^{2}+\left|v_{1}-v_{1}(p)\right|^{2}<\delta_{1}^{2}\right\}$ и $U_{p}=\left\{\left|u_{1}-u_{1}(p)\right|^{2}+\mid v_{1}-\right.$ $\left.\left.v_{1}(p)\right|^{2}<\delta_{2}^{2}\right\}$ радиусов $\delta_{1}$ и $\delta_{2}, 0<\delta_{1}<\delta_{2} \ll 1$, и через $\rho_{p}: \mathbb{C P}^{2} \rightarrow \mathbb{R}$ обозначим гладкую неотрицательную функцию такую, что $\rho_{p \mid V_{p}} \equiv 1$ и $\rho_{p \mid \mathbb{C P}^{2}} \backslash U_{p} \equiv 0$.

Чтобы построить требуемое вложение, выберем следующим образом два покрытия $\left\{U_{i}\right\}$ и $\left\{V_{i}\right\}$ плоскости $\mathbb{C P}^{2}$ открытыми множествами.

Для каждой точки $s_{i} \in \mathrm{Crit}_{\text {analytic }} \bar{H}$, не являющейся отрицательным ноудом, выберем небольшие окрестности $V_{s_{i}}^{\prime} \subset V_{s_{i}} \subset U_{s_{i}} \subset U_{s_{i}}^{\prime}$ такие, что:

$\left(c_{1}\right)$ кривая $U_{s_{i}}^{\prime} \cap \bar{H}$ является аналитической в $U_{s_{i}}^{\prime}$;

$\left(c_{2}\right)$ прообраз $\tilde{f}^{-1}\left(U_{s_{i}}^{\prime}\right)$ распадается в несвязное объединение окрестностей точек $y_{i, j} \in \tilde{f}^{-1}\left(s_{i}\right)$;

$\left(c_{3}\right)$ радиус шара $V_{s_{i}}^{\prime}\left(\right.$ соответственно, $U_{s_{i}}$ ) строго меньше радиуса шара $V_{s_{i}}$ (соответственно, $U_{s_{i}}^{\prime}$ ).

Пусть $V_{\infty}^{\prime} \subset V_{\infty} \subset U_{\infty} \subset U_{\infty}^{\prime}$ - открытые окрестности прямой $L_{\infty}$ такие, что:

$\left(c_{4}\right)$ в $U_{\infty}^{\prime}$ кривая Гурвица $\bar{H}$ совпадает с кривой $\bar{C}$, заданной в $U_{\infty}^{\prime}$ уравнением $v_{2}^{m}-1=0$

$\left(c_{5}\right) U_{\infty}^{\prime} \cap V_{s_{i}}^{\prime}=\varnothing$ для всех окрестностей $V_{s_{i}}^{\prime}$ критических точек $s_{i}$, выбранных вьше;

$\left(c_{6}\right) \bar{V}_{\infty}^{\prime} \subset V_{\infty}$ и $\bar{U}_{\infty} \subset U_{\infty}^{\prime}\left(\right.$ где $\bar{V}_{\infty}^{\prime}$, соответственно $\bar{U}_{\infty}$, является замыканием множества $V_{\infty}^{\prime}$, соответственно $\left.U_{\infty}\right)$.

Пусть $\rho_{\infty}: \mathbb{C P}^{2} \rightarrow \mathbb{R}$ - гладкая неотрицательная функция такая, что $\rho_{\infty} \mid V_{\infty} \equiv 1$ и $\rho_{\infty \mid \mathbb{C P}^{2} \backslash U_{\infty}} \equiv 0$. Добавим окрестности $U_{\infty}$ и $V_{\infty}$ к множествам выбранных выше окрестностей $\left\{U_{s_{i}}\right\}$ и $\left\{V_{s_{i}}\right\}$. Затем для каждой точки $p \in \mathbb{C}_{1}^{2} \backslash\left(\left(\bigcup V_{s_{i}}\right) \cup V_{\infty}\right)$ найдем открытые окрестности $V_{p} \subset U_{p}$ точки $p$ такие, что:

$\left(c_{7}\right) U_{p} \cap V_{s_{i}}^{\prime}=\varnothing$ и $U_{p} \cap V_{\infty}^{\prime}=\varnothing$ для всех выбранных выше окрестностей $V_{s_{i}}^{\prime}$ и $V_{\infty}^{\prime}$;

$\left(c_{8}\right)$ прообраз $\tilde{f}^{-1}\left(U_{p}\right)$ распадается в несвязное объединение окрестностей точек $y_{j} \in \tilde{f}^{-1}(p)$

$\left(c_{9}\right)$ если $p \in U_{s_{i}}^{\prime}$ (соответственно, $p \in U_{\infty}^{\prime}$ ) для некоторой выбранной выше окрестности $U_{s_{i}}^{\prime}$ (соответственно, $U_{\infty}^{\prime}$ ), то $U_{p} \subset U_{s_{i}}^{\prime}$ (соответственно, $U_{p} \subset U_{\infty}^{\prime}$ );

$\left(c_{10}\right)$ если $p \notin\left(\cup_{s_{i} \in \mathrm{Crit}_{\text {analytic }} \bar{H}} U_{s_{i}}^{\prime}\right) \cup U_{\infty}^{\prime}$, то $U_{p} \cap\left(\left(\cup_{s_{i} \in \mathrm{Crit}_{\text {analytic }}} \bar{H} U_{s_{i}}\right) \cup\right.$ $\left.U_{\infty}\right)=\varnothing$. 
Добавим окрестности $U_{p}$ и $V_{p}$ к выбранным выше множествам окрестностей $\left\{U_{s_{i}}\right\}$ и $\left\{V_{s_{i}}\right\}$ (здесь одно из $s_{i}$ равно бесконечности). В результате мы получим два открытых покрытия $\mathcal{V}=\left\{V_{p}\right\}$ и $\mathcal{U}=\left\{U_{p}\right\}$ проективной плоскости $\mathbb{C P}^{2}$.

Для $U^{\prime}=U_{\infty}^{\prime}$ пусть $\left(w_{3, \infty}, \ldots, w_{m_{\infty}, \infty}\right)$ - функции в $\tilde{Y} \backslash \tilde{f}^{-1}\left(L_{\infty}\right)$, определенные как $w_{j, \infty}=\tilde{f}^{*}\left(\rho_{\infty}\right) w_{j}^{\prime}$ для $j=3, \ldots, m_{\infty}$, где функции $w_{j}^{\prime}$ были определены в (3.1).

Для каждой окрестности $U_{s_{i}}^{\prime}$, где $s_{i} \in \mathrm{Crit}_{\text {analytic }} \bar{H}$, сушествуют комплексно-аналитические функции $w_{1, s_{i}}^{\prime}, \ldots, w_{m_{s_{i}}, s_{i}}^{\prime}$ в $\widetilde{U}_{s_{i}}^{\prime}=\tilde{f}^{-1}\left(U_{s_{i}}^{\prime}\right)$ такие, что эти функции вместе с $\tilde{f}^{*}\left(u_{1}\right)$ и $\tilde{f}^{*}\left(v_{1}\right)$ задают аналитическое вложение окрестности $\widetilde{U}_{s_{i}}^{\prime}$ в $\mathbb{C}^{m_{s_{i}}+2}$. Обозначим через $w_{j, s_{i}}=\tilde{f}^{*}\left(\rho_{s_{i}}\right) w_{j, s_{i}}^{\prime}, 1 \leqslant j \leqslant m_{s_{i}}$, функции на $\tilde{Y}$.

По построению открытых покрытий прообраз $\tilde{f}^{-1}\left(V_{p}\right)=\bigsqcup \widetilde{V}_{p, j}$ (соответственно, $\left.\tilde{f}^{-1}\left(U_{p}\right)=\bigsqcup \widetilde{U}_{p, j}\right)$ распадается в несвязное объединение $m_{p}=n_{p}$ связных окрестностей $\widetilde{V}_{p, j}\left(\right.$ соответственно, $\left.\widetilde{U}_{p, j}\right), j=1, \ldots, n_{p}$. Если $p \in \bar{H}$, то окрестность $\widetilde{U}_{p, j}$ изоморфна подмногообразию в $\mathbb{C}^{3}$, заданному в координатах $\left(u_{1}, v_{1}, w_{j, p}^{\prime}\right)$ уравнением

$$
\left(w_{j, p}^{\prime}-w_{j, p}^{0}\right)^{r_{j, p}}=v_{1}-v_{1, p}\left(u_{1}\right)
$$

где $v_{1}-v_{1, p}\left(u_{1}\right)=0$ - уравнение кривой $\bar{H}$ в $U_{p}$. Продолжим функции $w_{j, p}^{\prime}$, положив $w_{j, p \mid \widetilde{U}_{p, l}}^{\prime} \equiv 0$ для $l \neq j$, и выберем константы $w_{j, p}^{0}, j=1, \ldots, m_{p}$, так, что функции $\left(u_{1}, v_{1}, w_{1, p}^{\prime}, \ldots, w_{m_{p}, p}^{\prime}\right)$ задают гладкое вложение окрестности $\widetilde{U}_{p}$ в $\mathbb{C}^{m_{p}+2}$. Обозначим через $w_{j, p}=\tilde{f}^{*}\left(\rho_{p}\right) w_{j, p}^{\prime}, j=1, \ldots, m_{p}$, функции в $\tilde{Y}$.

Заметим, что если $y \in \tilde{f}^{-1}(\bar{H}) \cap \widetilde{V}_{p, j} \cap \widetilde{U}_{q, j}$, где $p, q \neq \infty$ и $p, q \notin \operatorname{Crit}_{\text {analytic }} \bar{H}$, то из определения функций $w_{j, p}$ и $w_{j, q}$ следует, что $r_{j, p}=r_{j, q}=r_{j}$ и сушествует корень $\zeta_{p, q}$ степени $r_{j}$ из единищы такой, что

$$
\begin{aligned}
w_{j, q} & =\rho_{q}\left(u_{1}, v_{1}\right)\left(\zeta_{p, q}\left(w_{j, p}-w_{j, p}^{0}\right)+w_{j, p}^{0}\right), \\
w_{j^{\prime}, q} & \equiv 0, \quad j^{\prime} \neq j,
\end{aligned}
$$

в некоторой окрестности точки $y$.

Аналогично, если $y \in \tilde{f}^{-1}(\bar{H}) \cap \widetilde{V}_{p, j} \cap \widetilde{U}_{q}$ (соответственно, если $y \in \tilde{f}^{-1}(\bar{H}) \cap$ $\left.\widetilde{V}_{q} \cap \widetilde{U}_{p, j}\right)$, где $q=\infty$ либо $q \in \operatorname{Sing}_{\text {analytic }} \bar{H}$, то из определения функций $w_{j, p}$ и $w_{i, q}$ из свойств $\left(c_{1}\right),\left(c_{4}\right),\left(c_{9}\right)$ и $\left(c_{10}\right)$ следует, что

$$
\left(w_{j, p}-w_{j, p}^{0}\right)^{r_{j}}=v_{1}-F\left(u_{1}\right)
$$

и

$$
w_{i, q}=\rho_{q}\left(u_{1}, v_{1}\right) h_{i}\left(u_{1}, v_{1}, w_{j, p}\right), \quad 1 \leqslant i \leqslant m_{q}
$$

(соответственно, $w_{j, p}=\rho_{p}\left(u_{1}, v_{1}\right) h_{j}\left(u_{1}, v_{1}, w_{1, q}, w_{2, q}, w_{3, q}, \ldots, w_{m_{q}, q}\right)$, и здесь $w_{1, q}$ и $w_{2, q}$ являются константами, если $q=\infty$ ), в некоторой окрестности точки $y$, где $F$ и все функции $h_{i}$ (соответственно, $h_{j}$ ) являются аналитическими функциями, a $v_{1}-F\left(u_{1}\right)=0$ - аналитическое уравнение одной из ветвей кривой $\bar{H}$. 
Если $p \notin \bar{H} \cup L_{\infty}$, то мы можем предполагать, что $\tilde{f}$ задает изоморфизмы окрестностей $\widetilde{U}_{p, j}$ и $U_{p}$ для $j=1, \ldots, m_{p}=N$. Выберем $N$ различных констант $w_{j, p}^{0}$ и определим функции $w_{j, p}=\tilde{f}^{*}\left(\rho_{p}\right) w_{j, p}^{\prime}, j=1, \ldots, m_{p}$, в $\tilde{Y}$, где $w_{j, p}^{\prime}$ - это функции, определенные в $\widetilde{U}_{p}$ следуюшим образом:

$$
w_{j, p}^{\prime}(q) \equiv \begin{cases}w_{j, p}^{0}, & \text { если } q \in \widetilde{U}_{p, j}, \\ 0, & \text { если } q \notin \widetilde{U}_{p, j} .\end{cases}
$$

Выберем конечное покрытие $\widetilde{\mathcal{V}}_{0}=\left\{\widetilde{V}_{p_{i}, j} \mid 1 \leqslant i \leqslant k, 1 \leqslant j \leqslant m_{p_{i}}\right\} \cup\left\{\widetilde{V}_{\infty}\right\}$ плоскости $\widetilde{Y}$ и положим

$$
M=m_{\infty}+\sum_{j=1}^{k} m_{p_{j}},
$$

$w_{3}=w_{3, \infty}, \ldots, w_{m_{\infty}}=w_{m_{\infty}, \infty}$. Перенумеруем множество функций

$$
\left\{w_{j, p_{i}} \mid 1 \leqslant i \leqslant k, 1 \leqslant j \leqslant m_{p_{i}}\right\}
$$

числами $m_{\infty}+1, \ldots, M$.

Рассмотри линейную проекцию $p: \mathbb{C P}^{M} \rightarrow \mathbb{C P}^{2}$, заданную формулой

$$
\left(z_{0}: z_{1}: z_{2}: \ldots: z_{M}\right) \rightarrow\left(z_{0}: z_{1}: z_{2}\right)
$$

Базисное множество проекции $p$ совпадает с проективным подпространством $P \simeq$ $\mathbb{C P}^{M-3}$, заданным уравнениями $z_{0}=z_{1}=z_{2}=0$. Ограничение проекции $p$ на $\mathcal{L}=\mathbb{C P}^{M} \backslash P$ определяет на $\mathcal{L}$ структуру векторного расслоения над $\mathbb{C P}^{2}$, нулевое сечение которого задано уравнениями $z_{3}=\cdots=z_{M}=0$. Над картами $\mathbb{C}_{i}^{2}$ с координатами $\left(u_{i}, v_{i}\right), i=1,2,3$, расслоение $\mathcal{L}$ является тривиальным и изомор фно произведению $\mathbb{C}_{i}^{M} \simeq \mathbb{C}_{i}^{2} \times \mathbb{C}_{i}^{M-2}$. В частности, $\left(z_{3} / z_{0}, \ldots, z_{M} / z_{0}\right)$ являются координатами в $\mathbb{C}_{1}^{M-2}$.

Над картой $\mathbb{C}_{1}^{2}$ рассмотрим отображение $\alpha^{\prime}: \tilde{f}^{-1}\left(\mathbb{C}_{1}^{2}\right) \rightarrow \mathbb{C}_{1}^{M}$, заданное формулой

$$
\alpha^{\prime}(y)=\left(\tilde{f}^{*}\left(u_{1}\right)(y), \tilde{f}^{*}\left(v_{1}\right)(y), w_{3}(y), \ldots, w_{M}(y)\right) .
$$

Поскольку для точки $p_{i} \neq \infty$ каждая окрестность $U_{p_{i}}$ содержится в $\mathbb{C}_{1}^{2}$ и все функции $w_{j, p_{i}}$ тождественно равны нулю в точках, лежащих над дополнением к $U_{p_{i}}$, то отображение $\alpha^{\prime}$ может быть продолжено до отображения $\alpha: \widetilde{Y} \rightarrow \mathcal{L}$ следующим образом. Над окрестностью $\tilde{f}^{-1}\left(V_{\infty}^{\prime}\right)$ отображение $\alpha$ совпадает с $i$ ○ $h$, где отображение $h$ было определено выше, а $i$ - это линейное вложение $\mathbb{C P}^{m}$ в $_{\mathbb{C P}}{ }^{M}$, заданное формулой

$$
i\left(\left(z_{0}: \ldots: z_{m_{\infty}}\right)\right)=\left(z_{0}: \ldots: z_{m_{\infty}}: 0: \ldots: 0\right)
$$

Легко видеть, что $\alpha$ является вложением таким, что $\alpha\left(\widetilde{V}_{s_{i}}^{\prime}\right)$ и $\alpha\left(\widetilde{V}_{\infty}^{\prime}\right)$ - аналитические множества $\mathcal{L}$ для $\widetilde{V}_{\infty}^{\prime}=\tilde{f}^{-1}\left(V_{\infty}^{\prime}\right)$ и для всех окрестностей $\widetilde{V}_{s_{i}}^{\prime}=\tilde{f}^{-1}\left(V_{s_{i}}^{\prime}\right)$, $s_{i} \in$ Crit $_{\text {analytic }} \bar{H}$. 
Обозначим через $\Omega$ ограничение формы Фубини-Штуди $\Omega_{M}$ на $\mathcal{L}$. В карте $\mathbb{C}_{1}^{M}$ она имеет следуюший вид:

$$
\Omega=\frac{i \sum_{k=1}^{M}\left(d w_{k} \wedge d \bar{w}_{k}+\sum_{j \neq k}\left(\bar{w}_{j} w_{j} d w_{k} \wedge d \bar{w}_{k}-\bar{w}_{j} w_{k} d w_{j} \wedge d \bar{w}_{k}\right)\right)}{\left(1+\sum_{j=1}^{M} \bar{w}_{j} w_{j}\right)^{2}}
$$

где $w_{k}=\frac{z_{k}}{z_{0}}$ и $w_{1}=u_{1}, w_{2}=v_{1}$.

Обозначим одинаково набор из двух положительных чисел $\bar{\varepsilon}=\left(\varepsilon_{1}, \varepsilon_{2}\right)$ и линейное преобразование $\bar{\varepsilon}: \mathbb{C P}^{M} \rightarrow \mathbb{C P}^{M}$, заданное формулой

$$
\left(z_{0}: z_{1}: z_{2}: z_{3}: \ldots: z_{M}\right) \rightarrow\left(z_{0}: z_{1}: \varepsilon_{1} z_{2}: \varepsilon_{2} z_{3}: \ldots: \varepsilon_{2} z_{M}\right) .
$$

Обозначим через $\omega_{\bar{\varepsilon}}$ ограничение формы $\Omega$ на $\tilde{Y}_{\bar{\varepsilon}}=(\bar{\varepsilon} \circ \alpha)(\tilde{Y})$. Покажем, что существуют положительная константа $c_{1}$ и положительная функция $c_{2}(t), t \in$ $\left(0, c_{1}\right]$, такие, что $\widetilde{Y}_{\bar{\varepsilon}}$ является симплектическим подмногообразием в $\mathcal{L}$ для всех $\bar{\varepsilon}=\left(\varepsilon_{1}, \varepsilon_{2}\right)$ при $\varepsilon_{1} \leqslant c_{1}$ и $\varepsilon_{2} \leqslant c_{2}\left(\varepsilon_{1}\right)$. Заметим, что если $\varepsilon_{1}$ достаточно мало, то образ кривой $\bar{H}$ при отображении $\left(z_{0}: z_{1}: z_{2}\right) \rightarrow\left(z_{0}: z_{1}: \varepsilon_{1} z_{2}\right)$ является симплектическим.

Для каждого $\bar{\varepsilon}$ форма $\omega_{\bar{\varepsilon}}$ является симплектической в точках, принадлежащих окрестностям $(\bar{\varepsilon} \circ \alpha)\left(\widetilde{V}_{s_{i}}^{\prime}\right)$, где $s_{i}$ - аналитические критические точки кривой $\bar{H}$, и в точках из $(\bar{\varepsilon} \circ \alpha)\left(\widetilde{V}_{\infty}^{\prime}\right)$, так как $\tilde{Y}_{\bar{\varepsilon}}$ является аналитическим подмногообразием многообразия $\mathcal{L}$ в этих точках.

Рассмотрим точку $y \in \tilde{f}^{-1}(\bar{H})$, принадлежащую точкам ветвления отображения $\tilde{f}$ и такую, что $\tilde{f}(y) \notin \widetilde{U}_{p}$ для $p \in \operatorname{Crit}_{\text {analytic }} \bar{H}$ и $p=\infty$. Из (3.2) следует, что после перенумерации координат $w_{3}, \ldots, w_{M}$ мы можем предполагать, что $\widetilde{Y}$ задается в окрестности точки $\alpha(y)$ уравнениями

$$
\begin{gathered}
\left(w_{3}-w_{3,0}\right)^{r}=v_{1}-F\left(u_{1}\right), \\
w_{j}=\rho_{j}\left(u_{1}, v_{1}\right) h_{j}\left(w_{3}\right), \quad j \geqslant 4,
\end{gathered}
$$

где $r \geqslant 2, \rho_{j}$ - гладкие функции, $h_{j}=\zeta_{3, j}\left(w_{3}-w_{3,0}\right)+w_{j, 0}-$ аналитические функции (здесь либо $\zeta_{3, j}-$ корень степени $r$ из единицы, либо $\left.\zeta_{3, j}=0\right), v_{1}-F\left(u_{1}\right)=0$ уравнение ветви кривой $\bar{H}$ в точке $\tilde{f}(y)$ и $\alpha(y)=\left(u_{1,0}, F\left(u_{1,0}\right), w_{3,0}, \ldots, w_{M, 0}\right)$. Тогда многообразие $\widetilde{Y}_{\bar{\varepsilon}}$ задается уравнениями

$$
\begin{aligned}
& \varepsilon_{1}\left(w_{3}-\varepsilon_{2} w_{3,0}\right)^{r}=\varepsilon_{2}^{r}\left(v_{1}-\varepsilon_{1} F\left(u_{1}\right)\right), \\
& w_{j}=\varepsilon_{2} \rho_{j}\left(u_{1}, \frac{v_{1}}{\varepsilon_{1}}\right) h_{j}\left(\frac{w_{3}}{\varepsilon_{2}}\right), \quad j \geqslant 4,
\end{aligned}
$$

в окрестности точки $(\bar{\varepsilon} \circ \alpha)(y)=\left(u_{1,0}, \varepsilon_{1} F\left(u_{1,0}\right), \varepsilon_{2} w_{3,0}, \ldots, \varepsilon_{2} w_{M, 0}\right)$.

Обозначим $A_{1}=\frac{\partial F}{\partial u_{1}}(\alpha(y)), \quad A_{2}=\frac{\partial F}{\partial \bar{u}_{1}}(\alpha(y)), \quad B_{j}=\frac{\partial h_{j}}{\partial w_{3}}(\alpha(y)), \quad C_{j, 1}=$ $\frac{\partial \rho_{j}}{\partial u_{1}}(\alpha(y)), \quad C_{j, 2}=\frac{\partial \rho_{j}}{\partial \bar{u}_{1}}(\alpha(y)), \quad D_{j, 1}=\frac{\partial \rho_{j}}{\partial v_{1}}(\alpha(y)), D_{j, 2}=\frac{\rho_{j}}{\partial \bar{v}_{1}}(\alpha(y)), \rho_{j, 0}=$ $\rho_{j}(\alpha(y)), h_{j, 0}=h_{j}\left(w_{3,0}\right), j=4, \ldots, M$. Из (3.7) следует, что в точке $(\bar{\varepsilon} \circ \alpha)(y)$ имеет место

$$
\begin{aligned}
& d v_{1}=\varepsilon_{1}\left(A_{1} d u_{1}+A_{2} d \bar{u}_{1}\right), \\
& d \bar{v}_{1}=\varepsilon_{1}\left(\bar{A}_{2} d u_{1}+\bar{A}_{1} d \bar{u}_{1}\right),
\end{aligned}
$$


а также имеют место равенства

$$
\begin{aligned}
& d w_{j}=\rho_{j, 0} B_{j} d w_{3}+\varepsilon_{2} h_{j, 0}\left(C_{j, 1} d u_{1}+C_{j, 2} d \bar{u}_{1}+D_{j, 1} \frac{d v_{1}}{\varepsilon_{1}}+D_{j, 2} \frac{d \bar{v}_{1}}{\varepsilon_{1}}\right), \\
& d \bar{w}_{j}=\rho_{j, 0} \bar{B}_{j} d \bar{w}_{3}+\varepsilon_{2} \bar{h}_{j, 0}\left(\bar{C}_{j, 2} d \bar{u}_{1}+\bar{C}_{j, 1} d u_{1}+\bar{D}_{j, 2} \frac{d v_{1}}{\varepsilon_{1}}+\bar{D}_{j, 1} \frac{d \bar{v}_{1}}{\varepsilon_{1}}\right),
\end{aligned}
$$

$j=4, \ldots, M$.

Если мы подставим (3.8) в (3.9), то получим

$$
\begin{aligned}
& d w_{j}=\rho_{j, 0} B_{j} d w_{3}+\varepsilon_{2} \nu_{j}, \\
& d \bar{w}_{j}=\rho_{j, 0} \bar{B}_{j} d \bar{w}_{3}+\varepsilon_{2} \bar{\nu}_{j},
\end{aligned}
$$

где формы $\nu_{j}$ и $\bar{\nu}_{j}$ не зависят от $\bar{\varepsilon}$ при $j=4, \ldots, M$.

Из (3.8) и (3.10) следует, что для каждого достаточно малого $\bar{\varepsilon}$ касательное пространство к многообразию $\widetilde{Y}_{\bar{\varepsilon}}$ в точке $(\bar{\varepsilon} \circ \alpha)(y)$ очень близко к касательному пространству в точке $(\bar{\varepsilon} \circ \alpha)(y)$ алгебраического многообразия $Z$, заданного уравнениями $v_{1}=\varepsilon_{1} F\left(u_{1,0}\right), w_{j}-w_{j, 0}=\rho_{j, 0} B_{j}\left(w_{3}-w_{3,0}\right), j=4, \ldots, M$. Следовательно, для каждого очень малого $\bar{\varepsilon}$ форма $\omega_{\bar{\varepsilon}}$ является симплектической в точке $(\bar{\varepsilon} \circ \alpha)(y)$. По непрерывности она является симплектической в некоторой окрестности точки $(\bar{\varepsilon} \circ \alpha)(y)$.

Рассмотрим точку $y \in \tilde{f}^{-1}(\bar{H})$, принадлежащую к точкам ветвления отображения $\tilde{f}$ и такую, что $\tilde{f}(y) \in U_{p}$ для некоторого $p \in \operatorname{Crit}_{\text {analytic }} \bar{H}$ либо $p=\infty$. Из (3.3) и (3.4) следует, что после перенумерации координат $w_{3}, \ldots, w_{M}$ мы можем предполагать, что сушествует некоторое $n, 3 \leqslant n \leqslant M$, такое, что $\widetilde{Y}$ задается в окрестности точки $\alpha(y)$ уравнениями

$$
\begin{aligned}
& h_{j}\left(u_{1}, v_{1}, w_{3}, \ldots, w_{n}\right)=0, \quad j=3, \ldots, n, \\
& \rho_{j}\left(u_{1}, v_{1}\right) h_{j}\left(u_{1}, v_{1}, w_{3}, \ldots, w_{n}\right)=w_{j}, \quad j=n+1, \ldots, M,
\end{aligned}
$$

где $\rho_{j}$ - гладкие функции и $h_{j}-$ аналитические функции в точке $\tilde{f}(y)$. Пусть $\left(u_{1,0}, v_{1,0}, w_{3,0}, \ldots, w_{M, 0}\right)-$ координаты точки $\alpha(y)$. Тогда многообразие $\widetilde{Y}_{\bar{\varepsilon}}$ задается в окрестности точки $(\bar{\varepsilon} \circ \alpha)(y)=\left(u_{1,0}, \varepsilon_{1} v_{1,0}, \varepsilon_{2} w_{3,0}, \ldots, \varepsilon_{2} w_{M, 0}\right)$ уравнениями

$$
\begin{gathered}
h_{j}\left(u_{1}, \frac{v_{1}}{\varepsilon_{1}}, \frac{w_{3}}{\varepsilon_{2}}, \ldots, \frac{w_{n}}{\varepsilon_{2}}\right)=0, \quad j=3, \ldots, n, \\
\varepsilon_{2} \rho_{j}\left(u_{1}, \frac{v_{1}}{\varepsilon_{1}}\right) h_{j}\left(u_{1}, \frac{v_{1}}{\varepsilon_{1}}, \frac{w_{3}}{\varepsilon_{2}}, \ldots, \frac{w_{n}}{\varepsilon_{2}}\right)=w_{j}, \quad j=n+1, \ldots, M .
\end{gathered}
$$

Положим $A_{j, l}=\frac{\partial h_{j}}{\partial w_{l}}(\alpha(y))$ для $1 \leqslant j \leqslant M, 1 \leqslant l \leqslant n$ (здесь $w_{1}=u_{1}$ и $\left.w_{2}=v_{1}\right)$, и пусть $B_{j}=\rho_{j}\left(u_{1,0}, v_{1,0}\right)$ для $n+1 \leqslant j \leqslant M$. 
Из (3.12) следует, что для каждого фиксированного $\varepsilon_{1}$ и для достаточно малого $\varepsilon_{2}$ касательное пространство к $\widetilde{Y}_{\bar{\varepsilon}}$ в точке $(\bar{\varepsilon} \circ \alpha)(y)$ очень близко к касательному пространству в точке $(\bar{\varepsilon} \circ \alpha)(y)$ линейного многообразия $Z$, заданного уравнениями

$$
\begin{aligned}
\sum_{l=3}^{n} A_{j, l}\left(w_{l}-\varepsilon_{2} w_{l, 0}\right) & =-A_{j, 1}\left(u_{1}-u_{1,0}\right)-\frac{A_{j, 2}}{\varepsilon_{1}}\left(v_{1}-\varepsilon_{1} v_{1,0}\right), \quad 3 \leqslant j \leqslant n, \\
B_{j} \sum_{l=3}^{n} A_{j, l}\left(w_{l}-\varepsilon_{2} w_{l, 0}\right) & =w_{j}-\varepsilon_{2} w_{j, 0}, \quad n+1 \leqslant j \leqslant M .
\end{aligned}
$$

Следовательно, для фиксированного $\varepsilon_{1}$ и для достаточно малого $\varepsilon_{2}$ форма $\omega_{\bar{\varepsilon}}$ является симплектической в точке $(\bar{\varepsilon} \circ \alpha)(y)$. По непрерывности она является симплектической в некоторой окрестности точки $(\bar{\varepsilon} \circ \alpha)(y)$.

Наконец, для каждой точки $y$, не принадлежащей множеству ветвления отображения $\tilde{f}$, многообразие $\tilde{Y}$ локально задается в точке $\alpha(y)$ уравнениями $w_{j}=$ $F_{j}\left(u_{1}, v_{1}\right), j=3, \ldots, M$, где $F_{j}\left(u_{1}, v_{1}\right)$ - некоторые гладкие функции в окрестности точки $\tilde{f}(y)$. Следовательно, многообразие $\tilde{Y}_{\bar{\varepsilon}}$ локально задается в точке $(\bar{\varepsilon} \circ \alpha)(y)$ уравнениями $w_{j}=\varepsilon_{2} F_{j}\left(u_{1}, \frac{v_{1}}{\varepsilon_{1}}\right), j=3, \ldots, M$. Легко видеть, что для каждого фиксированного $\varepsilon_{1}$ и для достаточно малых $\varepsilon_{2}$ форма $\omega_{\bar{\varepsilon}}$ является симплектической в $(\bar{\varepsilon} \circ \alpha)(y)$, так как многообразие $\widetilde{Y}_{\bar{\varepsilon}}$ очень близко к алгебраическому многообразию, заданному уравнениями $w_{j}=0, j=3, \ldots, M$, которое является симплектическим.

Чтобы завершить доказательство, достаточно воспользоваться компактностью многообразия $\widetilde{Y}$.

Применяя лемму 2.1 и теорему Хиронаки о разрешении особенностей, получаем

СлЕДСТВИЕ 3.2. Пусть $\tilde{f}: \widetilde{Y} \rightarrow \mathbb{C P}^{2}$ - конечное накрытие, разветвленное вдоль кривой Гурвица $\bar{H}$ (возможнн, с отрицательными ноудами) и, возможнн, вдоль $L_{\infty}$, ассоциированное с монодромией $\mu: \pi_{1}\left(\mathbb{C}^{2} \backslash H\right) \rightarrow \Sigma_{N}$. Тогда существуют наборь $\left(M_{1}, \ldots, M_{k}\right) u\left(n_{1}, \ldots, n_{k}\right)$ положительных иелых чисел такие, что разрешение $\bar{Y}$ особенностей многообразия $\widetilde{Y}$ может бьть вложено как симплектическое подмногообразие в $\left(\mathbb{C P}^{M_{1}} \times \cdots \times \mathbb{C P}^{M_{k}}\right.$, $\left.\Omega_{n_{1}, \ldots, n_{k}}\right)$, где $\Omega_{n_{1}, \ldots, n_{k}}=n_{1} p_{1}^{*}\left(\Omega_{M_{1}}\right)+\cdots+n_{k} p_{k}^{*}\left(\Omega_{M_{k}}\right)$ u $\Omega_{M_{j}}-$ форма Фубини-ШImуди на $\mathbb{C P}^{M_{j}}$.

ТЕОРемА 3.3. В обозначениях теоремы 3.1 пусть $\widetilde{Y}$ является гладким многообразием. Тогда симплектическая структура, заданная симплектической формой $\omega_{\bar{\varepsilon}}$, построенной в доказательстве теоремы 3.1 , не зависит ни от $\bar{\varepsilon}$, если координать в $\bar{\varepsilon}$ являются достаточно небольиими, ни от вьбора покрытий $\mathcal{U}$ и $\mathcal{V}$, ни от выбора функиий $w_{i, j}$.

Кроме того, если $i: \widetilde{Y} \hookrightarrow \mathbb{C P}^{N}$ является алгебраическим вложением (в случае, когда $\bar{H}$ - алгебраическая кривая) $u \tilde{f}=p \circ i$, где $p: \mathbb{C P}^{N} \rightarrow \mathbb{C P}^{2}-$ линейная проекция, то $\left(\tilde{Y}, \omega_{\bar{\varepsilon}}\right)$ u $\left(\widetilde{Y}, i^{*}\left(\Omega_{N}\right)\right)$ симплектоморфнь при достаточно малых $\bar{\varepsilon}$, где $\Omega_{N}-$ форма Фубини-Штудина $\mathbb{C P}^{N}$ и $\omega_{\bar{\varepsilon}}-$ форма, построенная в доказательстве теоремы 3.1 . 
ДокАЗАТЕльСтво. Пусть $\omega_{\bar{\varepsilon}}=(\bar{\varepsilon} \circ \alpha)^{*}(\Omega)$, где вложение $\alpha: \widetilde{Y} \rightarrow \mathcal{L}$ было построено в доказательстве теоремы 3.1. Очевидно, формы $\omega_{\bar{\varepsilon}}$ гладко зависят от $\bar{\varepsilon}$. Заметим, что для каждого $\bar{\varepsilon}, 0<\varepsilon_{1} \leqslant c_{1}$ и $0<\varepsilon_{2} \leqslant c_{2}\left(\varepsilon_{1}\right)$, класс $\left[\omega_{\bar{\varepsilon}}\right] \in H^{2}(\widetilde{Y}, \mathbb{Z})$ является двойственным к классу $\left[\tilde{f}^{-1}(L)\right] \in H_{2}(\widetilde{Y}, \mathbb{Z})$, где $L$ - некоторая прямая в $\mathbb{C P}^{2}$. Следовательно, согласно теореме Мозера о стабильности симплектических структур (см. [16, теорема 3.17$]$ ) формы $\omega_{\bar{\varepsilon}}$ определяют одну и ту же симплектическую структуру, если $0<\varepsilon_{1} \leqslant c_{1}$ и $0<\varepsilon_{2} \leqslant c_{2}\left(\varepsilon_{1}\right)$.

Покажем, что симплектическая структура на $\widetilde{Y}$, задаваемая формами $\omega_{\bar{\varepsilon}}$, не зависит от выбора покрытий $\left\{U_{i}\right\}$ и $\left\{V_{i}\right\}$ и не зависит от выбора функций $w_{i, j}$, определяюших вложение $\alpha$. Действительно, пусть два набора $\left\{w_{i, j}^{\prime}\right\}$ и $\left\{w_{i, j}^{\prime \prime}\right\}$ функций задают два вложения $\alpha^{\prime}: \widetilde{Y} \rightarrow \mathcal{L}^{\prime} \subset \mathbb{C P}^{M^{\prime}}$ и $\alpha^{\prime \prime}: \widetilde{Y} \rightarrow \mathcal{L}^{\prime \prime} \subset \mathbb{C P}^{M^{\prime \prime}}$. Положим

$$
\omega_{\bar{\varepsilon}}^{\prime}=\left(\bar{\varepsilon} \circ \alpha^{\prime}\right)^{*}\left(\Omega^{\prime}\right), \quad \omega_{\bar{\varepsilon}}^{\prime \prime}=\left(\bar{\varepsilon} \circ \alpha^{\prime \prime}\right)^{*}\left(\Omega^{\prime \prime}\right),
$$

где $\Omega^{\prime}$ и $\Omega^{\prime \prime}$ - симплектические формы Фубини-Штуди на $\mathbb{C P}^{M^{\prime}}$ и $\mathbb{C P}^{M^{\prime \prime}}$ соответственно. В этом случае мы имеем вложение $\alpha^{\prime} \times \alpha^{\prime \prime}: \widetilde{Y} \rightarrow \mathcal{L}^{\prime} \times_{\mathbb{C P}^{2}} \mathcal{L}^{\prime \prime}$. Заметим, что форма $\Omega_{t}=t\left(p^{\prime}\right)^{*}\left(\Omega^{\prime}\right)+(1-t)\left(p^{\prime \prime}\right)^{*}\left(\Omega^{\prime \prime}\right)$ является кэлеровой формой для каждого $t \in[0,1]$. Поскольку отрезок $[0,1]$ является компактом, применяя вычисления, аналогичные вычислениям, проведенным в доказательстве теоремы 3.1 , легко показать, что сушествует $\bar{\varepsilon}=\bar{\varepsilon}^{\prime}=\bar{\varepsilon}^{\prime \prime}, 0<\varepsilon_{1} \leqslant \min \left(c_{1}^{\prime}, c_{1}^{\prime \prime}\right)$, $0<\varepsilon_{2} \leqslant \min \left(c_{2}^{\prime}\left(\varepsilon_{1}\right), c_{2}^{\prime \prime}\left(\varepsilon_{1}\right)\right)$, такое, что $\omega_{t, \bar{\varepsilon}}=\left(\bar{\varepsilon} \circ\left(\alpha^{\prime} \times \alpha^{\prime \prime}\right)\right)^{*}\left(\Omega_{t}\right)$ является симплектической формой на $\widetilde{Y}$ для всех $t \in[0,1]$. С другой стороны, $\omega_{0, \bar{\varepsilon}}=\omega_{\bar{\varepsilon}}^{\prime}$, $\omega_{1, \bar{\varepsilon}}=\omega_{\bar{\varepsilon}}^{\prime \prime}$ и формы $\omega_{t, \bar{\varepsilon}}$ принадлежат одному и тому же классу когомологий. Следовательно, по теореме Мозера о стабильности симплектических структур формы $\omega_{t, \bar{\varepsilon}}$ определяют одну и ту же симплектическую структуру на $\widetilde{Y}$.

В случае алгебраического вложения $i: \widetilde{Y} \hookrightarrow \mathbb{C P}^{N}$ такого, что $\tilde{f}=p \circ i$, где $p: \mathbb{C P}^{N} \rightarrow \mathbb{C P}^{2}-$ линейная проекция, обозначим через $\alpha^{\prime}: \widetilde{Y} \rightarrow \mathcal{L}^{\prime} \subset \mathbb{C P}^{M^{\prime}}$ одно из вложений, построенных в доказательстве теоремы 3.1, и положим $\alpha^{\prime \prime}=i$. Применяя те же аргументы, что и выше, получим, что $\left(\widetilde{Y}, \omega_{\bar{\varepsilon}}\right)$ и $\left(\widetilde{Y}, i^{*}\left(\Omega_{N}\right)\right)$ симплектоморфны для достаточно малых $\bar{\varepsilon}$, так как симплектические многообразия $\left(\widetilde{Y}, \omega_{\bar{\varepsilon}}^{\prime \prime}\right)$ симплектоморфны для всех положительных наборов $\bar{\varepsilon}$, где $\omega_{\bar{\varepsilon}}^{\prime \prime}=\left(\bar{\varepsilon} \circ \alpha^{\prime \prime}\right)^{*}\left(\Omega_{N}\right)$.

\section{§4. Вложения циклических накрытий плоскости} в рациональные проективные трехмерные многообразия

В этом параграфе мы используем предположения и обозначения из $§ 1$.

Пусть $\bar{H}$ - кривая Гурвища степени $m$. Рассмотрим бесконечное циклическое накрытие $f=f_{\infty}: X_{\infty} \rightarrow X^{\prime}=\mathbb{C}^{2} \backslash H$, ассоциированное с эпиморфизмом $\nu: \pi_{1}\left(\mathbb{C}^{2} \backslash H\right) \rightarrow \mathbb{F}_{1}$. Накрытие $f_{\infty}$ может быть пропущено через циклическое накрытие $f_{n}: X_{n}^{\prime} \rightarrow \mathbb{C}^{2} \backslash H$, ассоциированное с эпиморфизмом $\bmod _{n} \circ \nu$, $f_{\infty}=g_{n} \circ f_{n}$.

В этом параграфе мы покажем, что накрытие $f_{n}$ может быть продолжено до отображения $\bar{f}_{n}: \bar{X}_{n} \rightarrow \mathbb{C P}^{2}$, разветвленного вдоль $\bar{H}$ и, возможно, вдоль $L_{\infty}$ (если $n$ не является делителем $\operatorname{deg} \bar{H}$, то $\bar{f}_{n}$ разветвлено вдоль $L_{\infty}$ ), где $\bar{X}_{n}-$ некоторое компактное вешественное четырехмерное многообразие. 
Tеорема 4.1. Разрешение $\bar{X}_{n}$ особенностей ииклического накрытия проективной плоскости $\mathbb{C P}^{2}$ степени $n$, разветвленного вдоль кривой Гурвица $\bar{H}$ $u$, возможно, вдоль $L_{\infty}$, может быть вложено в некоторое рациональное проективное комплексное трехмерное многообразие (снабженное челочисленной кәлеровой симплектической структурой) как симплектическое подмногообразие.

ДокАЗАТЕЛЬСтво. Поскольку $\mathbb{C}^{2} \backslash H_{1}$ и $\mathbb{C}^{2} \backslash H_{2}$ диффеоморфны для $H$-изотопных кривых Гурвица $\bar{H}_{1}$ и $\bar{H}_{2}$, мы будем предполагать, что $\bar{H}$ удовлетворяет условиям $(*)$ и $(* *)$.

Из леммы 2.1 и теоремы Хиронаки о разрешении особенностей следует, что для доказательства теоремы достаточно показать, что для некоторого продолжения $\tilde{f}_{n}: \widetilde{X}_{n} \rightarrow \mathbb{C P}^{2}$ отображения $f_{n}^{\prime}: X_{n}^{\prime} \rightarrow X^{\prime}$ многообразие $\widetilde{X}_{n}$ может быть вложено в проективное комплексное рациональное трехмерное многообразие (снабженное целочисленной кэлеровой симплектической структурой) как симплектическое подмногообразие с аналитическими особенностями.

Чтобы показать это, обозначим через $d$ наименьшее неотрицательное целое число такое, что $m+d$ делится нацело на $n$. Положим $m+d=k n$ и рассмотрим линейное расслоение $p: \mathcal{L}(k) \rightarrow \mathbb{C P}^{2}($ см. $\S 1)$, ассоциированное с пучком $\mathcal{O}_{\mathbb{C P}^{2}}(k)$. Согласно лемме 1.4 кривая Гурвица $\bar{H}$ совпадает с множеством нулей гладкого сечения $\bar{s}_{m}$ расслоения $\mathcal{L}(m)$ над $\mathbb{C P}^{2}$ таким, что $\bar{s}_{m}$ является аналитическим в некоторой окрестности $U$ прямой $L_{\infty}$ и в некоторых окрестностях всех критических точек кривой $\bar{H}$.

Обозначим через $\bar{s}_{d}$ сечение расслоения $\mathcal{L}(d)$, заданного над $\mathbb{C}_{2}^{2}$ функцией $w_{2}=u_{2}^{d}$. Произведение

$$
\bar{s}_{m+d}=\bar{s}_{m} \bar{s}_{d}
$$

является сечением расслоения $\mathcal{L}(m+d)$, где $\bar{s}_{m}$ - это сечение расслоения $\mathcal{L}(m)$, удовлетворяюшее всем условиям из леммы 1.4 .

Определим $\alpha: \widetilde{X}_{n} \hookrightarrow \mathcal{L}(k)$ с помощью уравнения

$$
w_{i}^{n}=\bar{s}_{m+d}\left(u_{i}, v_{i}\right)
$$

и положим $\tilde{f}_{n}=p_{\mid X_{n}}$, где $p: \mathcal{L}(k) \rightarrow \mathbb{C P}^{2}-$ морфизм, задаюший на $\mathcal{L}(k)$ структуру линейного расслоения. В частности, $\widetilde{X}_{n}$ задается уравнением

$$
w_{1}^{n}=F_{1}\left(u_{1}, v_{1}\right)
$$

в карте $\mathbb{C}_{1}^{3}$ и задается уравнением

$$
w_{2}^{n}=u_{2}^{d} F_{2}\left(u_{2}, v_{2}\right)
$$

в карте $\mathbb{C}_{2}^{3}$.

Очевидно, над $\mathbb{C P}^{2} \backslash\left(\bar{H} \cup L_{\infty}\right)$ накрытие $\tilde{f}_{n}$ является неразветвленным $n$-листным циклическим накрытием. Затем, все особые точки многообразия $\widetilde{X}_{n}$ лежат над особыми точками кривой $\bar{H}$ и, возможно, над $L_{\infty}$. Более того, по построению 
сечения $\bar{s}_{m+d}$ множество Sing $\widetilde{X}_{n}$ является комплексно-аналитическим в некоторой окрестности $U \subset \mathcal{L}(k)$.

Линейное расслоение $\mathcal{L}(k)$ является квазипроективньм многообразием, и при добавлении сечения "в бесконечности" оно может быть компактифицировано до проективного трехмерного рационального многообразия $\overline{\mathcal{L}}(k)$.

Многообразие $\overline{\mathcal{L}}(k)$ имеет много различных вложений в проективные пространства, так как группа Пикара $\operatorname{Pic}(\overline{\mathcal{L}}(k)) \simeq \mathbb{Z} \oplus \mathbb{Z}$. Мы можем выбрать одно из них, например следуюшее вложение.

В окрестности $\mathbb{C}_{1}^{3}$ с координатами $\left(u_{1}, v_{1}, w_{1}\right)$ рассмотрим мономы $u_{1}^{a_{1}} v_{1}^{a_{2}} w_{1}^{a_{3}}$, $0 \leqslant a_{1}+a_{2}+k a_{3} \leqslant k+1$, число которых равно $\frac{(k+2)(k+3)}{2}+3$. Положим $N=\frac{(k+2)(k+3)}{2}+2$ и рассмотрим рациональное отображение $h: \overline{\mathcal{L}}(k) \rightarrow \mathbb{C P}^{N}$, заданное в $\mathbb{C}_{1}^{3}$ функциями $z_{\bar{a}}=u_{1}^{a_{1}} v_{1}^{a_{2}} w_{1}^{a_{3}}$, где $\bar{a}=\left(a_{1}, a_{2}, a_{3}\right)$ - тройки целых чисел и $z_{\bar{a}}$ - однородные координаты в $\mathbb{C P}^{N}$. Легко проверяется, что $h$ является вложением.

Рассмотрим форму Фубини-Штуди $\Omega_{N}$ на $\mathbb{C P}^{N}$. Обозначим через $\Omega=h^{*}\left(\Omega_{N}\right)$ ее прообраз.

Как и в доказательстве теоремы 3.1 , используем одно и то же обозначение $\bar{\varepsilon}=$ $\left(\varepsilon_{1}, \varepsilon_{2}\right)$ для набора двух положительных чисел и для автоморфизма расслоения $\overline{\mathcal{L}}(k)$, заданного в $\mathbb{C}_{1}^{3}$ формулой $\left(u_{1}, v_{1}, w_{1}\right) \rightarrow\left(u_{1}, \varepsilon_{1} v_{1}, \varepsilon_{2} w_{1}\right)$.

Вычисления (мы опускаем их), аналогичные тем, которые были проведены в доказательстве теоремы 3.1 , показывают, что сушествуют положительная константа $c_{1}$ и положительная функция $c_{2}(t)$ такие, что $\widetilde{X}_{\bar{\varepsilon}}=(\bar{\varepsilon} \circ \alpha)\left(X_{n}\right)$ является симплектическим подмногообразием расслоения $\mathcal{L}$ с аналитическими особенностями для всех $\bar{\varepsilon}=\left(\varepsilon_{1}, \varepsilon_{2}\right)$ таких, что $\varepsilon_{1} \leqslant c_{1}$ и $\varepsilon_{2} \leqslant c_{2}\left(\varepsilon_{1}\right)$.

\section{§5. Многочлены Александера гурвицевских $C$-групп}

Пусть $\bar{H}$ - кривая (соответственно, топологическая кривая) Гурвица степени $m$. Поскольку копредставление Зариского-ван Кампена группы $\pi_{1}\left(\mathbb{C}^{2} \backslash H\right)$ является $C$-копредставлением гурвицевской $C$-группы степени $m$, то теоремы 0.1 и 0.2 являются следствиями следуюших теорем 5.1 и 5.2 .

TeOpema 5.1. Пусть $G \in \mathcal{H}$ - гурвицевская $C$-группа степени $m$ и $\Delta(t)-$ ее многочлен Александера. Тогда:

(i) $\Delta(t) \in \mathbb{Z}[t]$;

(ii) $\Delta(0)= \pm 1$;

(iii) корни многочлена $\Delta(t)$ являются корнями степени $m$ из единицы;

(iv) ранг свободной части абелевой группь $N^{\prime} / N^{\prime \prime}$ равен $\operatorname{deg} \Delta(t)$;

(v) действие $h_{\mathbb{C}}$ на $N / N^{\prime} \otimes \mathbb{C}$ полупросто.

ДокАЗАТЕЛЬСтво. Рассмотрим точную последовательность груп

$$
1 \rightarrow N \rightarrow G \stackrel{\nu}{\rightarrow} \mathbb{F}_{1} \rightarrow 1
$$

Эта точная последовательность индуцирует автоморфизм $\tilde{h} \in \operatorname{Aut} N$ (действие $C$-порождаюшего элемента $x \in \mathbb{F}_{1}$ на $N$ ) задано сопряжением $\tilde{h}(n)=\tilde{x}^{-1} n \tilde{x}$ для $n \in N$, где $\tilde{x}$ - это один из $C$-порождающих элементов группы $G$. Очевидно, 
автоморфизм $\tilde{h}$ определен однозначно с точностью до внутренних автоморфизмов групшы $N$, следовательно, $\tilde{h}$ определяет автоморфизм $h \in \operatorname{Aut} N / N^{\prime}$.

В работе [13] было доказано, что для гурвищевской $C$-групшы $G$ группа $N$ является конечно представимой. Следовательно, $N / N^{\prime}$ является конечно порожденной абелевой группой. Пусть $N / N^{\prime}=T \oplus F$ - разложение в прямую сумму группы кручения $T$ и свободной абелевой группы $F$. Отметим, что $T$ является конечной группой и $F-$ конечно порожденная группа. Автоморфизм $h$ группы $N / N^{\prime}$ индуцирует автоморфизм групшы $T$ и, следовательно, автоморфизм $\bar{h}$ групшы $F \simeq\left(N / N^{\prime}\right) / T$. Если мы выберем свободный над $\mathbb{Z}$ базис $\mathbb{Z}$-модуля $F$, то этот автоморфизм задается матрицей $H$ с целыми коэффициентами. Так как автоморфизм $h_{\mathbb{C}}$ векторного пространства $N / N^{\prime} \otimes \mathbb{C}$ может быть задан той же самой матрицей $H$, то многочлен $\Delta(t)=\operatorname{det}(H-t \mathrm{Id}) \in \mathbb{Z}[t]$. Поскольку $\bar{h} \in \operatorname{Aut} F$, то $\operatorname{det} \widetilde{H}= \pm 1$ и, следовательно, $\Delta(0)= \pm 1$.

Покажем, что $\tilde{h}^{m}$ является внутренним автоморфизмом группы $N$. Действительно, так как $G$ - гурвицевская $C$-группа степени $m$, то она порождается некоторыми $C$-порождающими элементами $x_{1}, \ldots, x_{m}$ такими, что произведение $x_{1} \ldots x_{m}$ принадлежит центру группы $G$, и поэтому $\tilde{x}^{m}=\tilde{n} \cdot x_{1} \ldots x_{m}$ для некоторого $\tilde{n} \in N$ задает на $N$ внутренний автоморфизм. Таким образом, индуцированные автоморфизмы $h^{m}$ группы $N / N^{\prime}$ и $h_{\mathbb{C}}^{m}$ векторного пространства $N / N^{\prime} \otimes \mathbb{C}$ являются тривиальньми, т.е. $h_{\mathbb{C}}^{m}=\mathrm{Id}$.

Поскольку $h_{\mathbb{C}}^{m}=\mathrm{Id}$, то все корни многочлена $\Delta(t)=\operatorname{det}\left(h_{\mathbb{C}}-t \mathrm{Id}\right)$ являются корнями степени $m$ из единицы и действие $h_{\mathbb{C}}$ на $N / N^{\prime} \otimes \mathbb{C}$ является полупростым.

Tеорема 5.2. Пусть $G$ - неприводимая гурвицевская $C$-группа. Тогда:

(i) $\Delta(1)=1$;

(ii) $\operatorname{deg} \Delta(t)$ является четным числом;

(iii) $\Delta(t)$ является возвратным многочленом.

ДокАЗАТЕЛЬСтво. Из работы $[9$, лемма 6] следует, что $\Delta(1)= \pm 1$. Покажем, что $t=-1$ также не является корнем многочлена $\Delta(t)$. Действительно, если $t=-1$ является корнем многочлена $\Delta(t)$, то $\Delta(t)=(t+1) P(t)$, где $P(t)-$ некоторый многочлен с целыми коэффициентами. Следовательно, $2 P(1)= \pm 1$, но это невозможно, так как $P(1)$ является цельм числом.

Согласно теореме 5.1 все корни многочлена $\Delta(t) \in \mathbb{Z}[t]$ являются корнями из единицы. Эти корни не являются вещественными числами, так как $t= \pm 1$ не являются корнями многочлена $\Delta(t)$. Следовательно, $\operatorname{deg} \Delta(t)$ является четным числом, и утверждение (ii) доказано.

Хорошо известно, что если $\lambda$ является примитивным корнем степени $k$ из единицы, $k>2$, и многочлен $P(t) \in \mathbb{Z}[t]$ имеет $\lambda$ в качестве одного из своих корней, то все примитивные корни степени $k$ из единицы являются корнями многочлена $P(t)$. В частности, $\lambda^{-1}$ также является корнем многочлена $P(t)$, и утверждение (iii) доказано.

Поскольку $\Delta(t)=\operatorname{det}\left(h_{\mathbb{C}}-t \mathrm{Id}\right)$ и $\operatorname{deg} \Delta(t)$ является четным числом, то $\Delta(t)=$ $t^{\operatorname{deg} \Delta(t)}+\ldots$ Пусть $\Delta(t)=\prod_{i} \Phi_{k_{i}}(t)$ - разложение в произведение $k_{i}$-х циклотомических многочленов. Поэтому утверждение (i) следует из хорошо известной леммы 5.3 , так как $\Delta(1)= \pm 1$. 
ЛЕмма 5.3. Пусть $\Phi_{k}(t)-k$-й ииклотомический многочлен, $k>1$. Тогда

$$
\Phi_{k}(1)=\left\{\begin{array}{l}
p, \quad \text { если } k=p^{n} \text { для некоторого простого числа } p \\
1, \quad \text { если } k \neq p^{n} \text { для любого простого числа } p .
\end{array}\right.
$$

ДОКАЗАТЕЛЬСТВО. Применяя индукцию по $k>1$, утверждение леммы следует из равенств

$$
\Phi_{k}(t)=\frac{\sum_{i=0}^{k-1} t^{i}}{\prod_{\substack{d \mid k \\ d<k}} \Phi_{d}(t)}, \quad \Phi_{k}(1)=\frac{k}{\prod_{\substack{d \mid k \\ d<k}} \Phi_{d}(1)}, \quad \Phi_{k}(0)=\frac{1}{\prod_{\substack{d \mid k \\ d<k}} \Phi_{d}(0)}
$$

СлЕДСТВИЕ 5.4. Пусть $G$ - неприводимая гурвицевская $C$-группа степени $m=p^{n}$, где $p$ - простое число. Тогда:

(i) $\Delta(t) \equiv 1$;

(ii) әруппа $G^{\prime} / G^{\prime \prime}$ является конечной абелевой группой.

ДоКАЗАТЕЛЬСТВо. Согласно теореме 5.1 все корни многочлена $\Delta(t)$ являются корнями степени $m$ из единицы. Пусть $\lambda$ - один из его корней. Предположим, что $\lambda$ является примитивным корнем степени $p^{k}$ из единицы, $1 \leqslant k \leqslant n$. Тогда $\lambda$ является корнем $p^{k}$-го циклотомического многочлена

$$
\Phi_{p^{k}}(t)=\sum_{i=0}^{p-1} t^{i p^{k-1}}
$$

и сушествует некоторый многочлен $f(t) \in \mathbb{Z}[t]$ такой, что $\Delta(t)=\Phi_{p^{k}}(t) f(t)$. Согласно теореме 5.2 значение $\Phi_{p^{k}}(1) f(1)= \pm 1$. С другой стороны, $f(1) \in \mathbb{Z}$ и $\Phi_{p^{k}}(1)=p$. Поэтому многочлен $\Delta(t)$ не имеет корней. Следовательно, $\operatorname{deg} \Delta(t)$ равна нулю и группа $G^{\prime} / G^{\prime \prime}$ не имеет свободной части, т. е. она является конечной абелевой группой.

Следствие 0.3 вытекает из следствия 5.4.

Лемма 5.5. Пусть $G_{1}, G_{2}-C$-группь $u \Delta_{1}(t), \Delta_{2}(t)-$ их многочлень Александера. Предположим, что существует $C$-эпиморфизм $f: G_{1} \rightarrow G_{2}$. Тогда многочлен $\Delta_{2}(t)$ является делителем многочлена $\Delta_{1}(t)$.

ДоказАТЕЛЬСтво. Обозначим через $N_{i}$ ядро канонического $C$-эпиморфизма $\nu_{i}: G_{i} \rightarrow \mathbb{F}_{1}, i=1,2$. Легко видеть, что гомоморфизм $g$ в коммутативной диаграмме

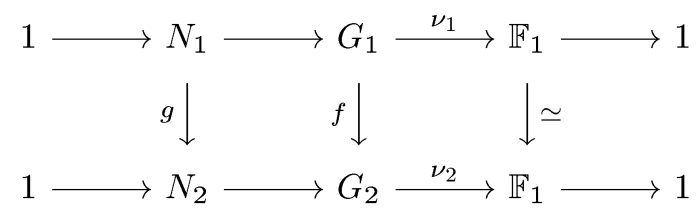


является эпиморфизмом. Эта диаграмма индуцирует следующую коммутативную диаграмму:

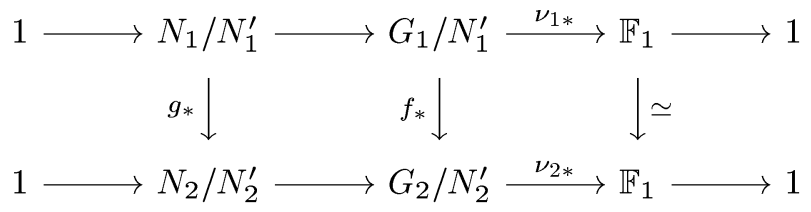

в которой $g_{*}$ также является эпиморфизмом.

Из диаграммы (5.1) следует, что $\Delta_{2}(t)$ является делителем многочлена $\Delta_{1}(t)$, так как $h_{2}\left(g_{*}(n)\right)=g_{*}\left(h_{1}(n)\right)$ для всех $n \in N_{1} / N_{1}^{\prime}$.

Теорема 5.6. Пусть $G$ - гурвицевская C-группа степени $m$. Тогда ее многочлен Александера $\Delta(t)$ делит многочлен $(t-1)\left(t^{m}-1\right)^{m-2}$.

ДокаЗАтельство. Рассмотрим гурвицевскую $C$-группу

$$
\widetilde{G}_{m}=\left\langle x_{1}, \ldots, x_{m} \mid\left[x_{i}, x_{1} \ldots x_{m}\right]=1, i=1, \ldots, m\right\rangle
$$

Для любой гурвицевской $C$-группы $G$ степени $m$ сушествует естественный $C$-эпиморфизм $f: \widetilde{G}_{m} \rightarrow G$, отображаюший $C$-порождаюшие элементы $x_{i}$ группы $\widetilde{G}_{m}$ в $C$-порождаюшие элементы $x_{i}$ группы $G$, произведение которых $x_{1} \ldots x_{m}$ принадлежит центру группы $G$. Следовательно, чтобы доказать теорему, достаточно показать, что многочлен Александера группы $\widetilde{G}_{m}$ равен $(-1)^{m-1}(t-1) \times$ $\left(t^{m}-1\right)^{m-2}$.

Обозначим через $\widetilde{N}_{m}$ ядро канонического гомоморфизма $\nu: \widetilde{G}_{m} \rightarrow \mathbb{F}_{1}$ и положим $y=x_{1} \ldots x_{m}$. Не ограничивая обшности, мы можем предполагать, что $\tilde{h}(n)=x_{m} n x_{m}^{-1}$ для $n \in \widetilde{N}_{m}$. В работе [13] при использовании метода Рейдемейстера-Шрайера было показано, что $\widetilde{N}_{m}$ порождается элементами

$$
a_{k, j}=x_{m}^{k} x_{j} x_{m}^{-(k+1)}
$$

где $j=2, \ldots, m-1, k \in \mathbb{Z}$, вместе с элементами

$$
a_{k, m}=x_{m}^{k} y x_{m}^{-(k+m)},
$$

где $k \in \mathbb{Z}$. В этом случае действие $\tilde{h}$ задается формулами $\tilde{h}\left(a_{k, j}\right)=a_{k+1, j}$.

Из соотношений $y x_{j}=x_{j} y, j=2, \ldots, m$, вытекают (см. [13]) соотношения

$$
a_{k, m}=a_{k+1, m}
$$

для $k \in \mathbb{Z}$ и соотношения

$$
a_{k, m} a_{k+m, j} a_{k+1, m}^{-1}=a_{k, j}
$$

для $j=2, \ldots, m-1$ и $k \in \mathbb{Z}$. Следовательно, $\tilde{N}_{m}$ является свободной группой, свободно порожденной элементами $a_{0, m}$ и $a_{k, j}, k=1, \ldots, m, j=2, \ldots, m-1$. 
Имеем $\tilde{h}\left(a_{0, m}\right)=a_{0, m}, \tilde{h}\left(a_{k, j}\right)=a_{k+1, j}$ для $k=1, \ldots, m-1, j=2, \ldots, m-1$ и $\tilde{h}\left(a_{m, j}\right)=a_{0, m}^{-1} a_{1, j} a_{0, m}$ для $j=2, \ldots, m-1$.

Пусть $\bar{a}_{k, j}$ - образ элемента $a_{k, j}$ в групше $\widetilde{N}_{m} / \widetilde{N}_{m}^{\prime}$. Тогда действие $h$ задается формулами

$$
\begin{aligned}
\tilde{h}\left(\bar{a}_{0, m}\right) & =\bar{a}_{0, m}, \\
h\left(\bar{a}_{k, j}\right) & =\bar{a}_{k+1, j}, \quad k=1, \ldots, m-1, \quad j=2, \ldots, m-1, \\
h\left(\bar{a}_{m, j}\right) & =\bar{a}_{1, j}, \quad j=2, \ldots, m-1 .
\end{aligned}
$$

С помощью простых вычислений легко показать, что характеристический многочлен автоморфизма $h$ равен $(-1)^{m-1}(t-1)\left(t^{m}-1\right)^{m-2}$.

Легко видеть [9, лемма 4], что для любой $C$-группы $G$ группа $G / G^{\prime}$ является конечно порожденной свободной абелевой группой. Более того, канонический эпиморфизм аb: $G \rightarrow G / G^{\prime}$ является $C$-гомоморфизмом, если мы выберем $\mathrm{ab}\left(x_{i}\right)$ в качестве $C$-порождающих элементов группы $G / G^{\prime}$, где $\left\{x_{i}\right\}$ - множество всех $C$-порождающих элементов групшы $G$. Скажем, что $C$-группа $G$ cocmoum из $n$ неприводимых компонент, если $G / G^{\prime} \simeq \mathbb{Z}^{n}$. Введение понятия числа неприводимых компонент гурвищевской $C$-группы объясняет следующая простая лемма (случай произвольной $C$-групшы см. в [10]).

ЛЕмма 5.7. Кривая (соответственно, топологическая кривая) Гурвица $\bar{H}$ состоит из $n$ неприводимых компонент тогда и только тогда, когда $\pi_{1}=\pi_{1}\left(\mathbb{C}^{2} \backslash H\right)$ состоит из $n$ неприводимых компонент.

С помощью простых вычислений легко показать, что многочлен Александера $\Delta(t)$ абелевой $C$-группы $\mathbb{Z}^{n}$ равен $(-1)^{n-1}(t-1)^{n-1}$. Следовательно, лемма 5.5 влечет следуюшую лемму.

Лемма 5.8. Многочлен Александера $\Delta(t)$ гурвичевской $C$-группы $G$, состоящей из $n$ неприводимых компонент, делится на $(t-1)^{n-1}$.

TеОРема 5.9. Пусть гурвицевская $C$-группа $G$ состоит из $n$ неприводимых компонент, и пусть $\Delta(t)$-ее многочлен Александера. Тогда

$$
\Delta(t)=(t-1)^{n-1} P(t),
$$

где $P(t) \in \mathbb{Z}[t]$ - некоторый многочлен, удовлетворяющий условию $P(1) \neq 0$.

ДокАЗАТЕЛЬСтво. Пусть гурвицевская $C$-группа $G$ имеет степень $m$. Чтобы получить некоторое $C$-копредставление группы $G$, достаточно добавить несколько $C$-соотношений в копредставление (5.2).

Поскольку $G$ состоит из $n$ неприводимых компонент, то множество $\{1, \ldots, m\}$ распадается в несвязное объединение $n$ подмножеств $J_{1}, \ldots, J_{n}$ таких, что $j_{1}, j_{2} \in J_{k}$ тогда и только тогда, когда $x_{j_{1}}$ и $x_{j_{2}}$ сопряжень в группе $G$. Пусть $J_{k}=\left\{j_{1, k}<\cdots<j_{m_{k}, k}\right\}$. Не ограничивая обшности, можем предполагать, что среди добавленных $C$-соотношений имеются соотношения

$$
x_{j_{i, k}} w_{j_{i, k}, j_{i+1, k}, 1}=w_{j_{i, k}, j_{i+1, k}, 1} x_{j_{i+1, k}}
$$


для $i=1, \ldots, m_{k}-1, k=1, \ldots, n$, и для некоторых слов $w_{j_{i, k}, j_{i+1, k}, 1}$. Пусть $\nu\left(w_{j_{i, k}, j_{i+1, k}, 1}\right)=x^{t_{j, k}}$, где $x-C$-порождаюший элемент группы $\mathbb{F}_{1}$.

Рассмотрим диаграмму (5.1), в которой $G_{1}=\widetilde{G}_{m}$ и $G_{2}=G$. В обозначениях из доказательства теоремы 5.6 элементы $\bar{a}_{0, m}$ и $\sum_{k=1}^{m} \bar{a}_{k, j}, j=2, \ldots, m-1$, порождают подгруппу

$$
\left(N_{1} / N_{1}^{\prime}\right)_{1}=\left\{y \in N_{1} / N_{1}^{\prime} \mid h(y)=y\right\}
$$

групшы $N_{1} / N_{1}^{\prime}$ и подпространство $\left(N_{1} / N_{1}^{\prime} \otimes \mathbb{C}\right)_{1}$, порожденное собственными векторами автоморфизма $h_{\mathbb{C}}$ с собственным значением 1 . Образ $g_{*}\left(\left(N_{1} / N_{1}^{\prime}\right)_{1}\right) \otimes \mathbb{C}$ совпадает с собственным подпространством пространства $N_{2} / N_{2}^{\prime} \otimes \mathbb{C}$, соответствующим собственному значению 1.

Применяя метод Рейдемейстера-Шрайера, можно показать, что элемент $a_{0, m}$, определенный в (5.4), вместе с элементами $a_{k, j}, k=1, \ldots, m, j=2, \ldots, m-1$, определенными в (5.3) (более точно, их образы при отображении $g_{*}$ ), также порождают группу $N_{2}$, и каждое соотношение (5.7) (после подстановки $x_{1}=$ $\left.y\left(x_{2} \ldots x_{m}\right)^{-1}\right)$ дает соотношения

$$
a_{r, j_{i, k}} \bar{w}_{r+1, j_{i, k}, j_{i+1, k}}=\bar{w}_{r, j_{i, k}, j_{i+1, k}} a_{r+t_{j_{i, k}}, j_{i+1, k}},
$$

если $1<j_{i, k}<j_{i+1, k}<m$,

$$
a_{0, m}\left(\prod_{s=2}^{m-1} a_{r+m-s, m+1-s}^{-1}\right) \bar{w}_{r+1,1, j_{i+1, k}}=\bar{w}_{r, 1, j_{i+1, k}} a_{r+t_{1, j_{i+1, k}}},
$$

если $1=j_{i, k}<j_{i+1, k}<m$,

$$
a_{r, j_{i, k}} \bar{w}_{r+1, j_{i, k}, m}=\bar{w}_{r, j_{i, k}, m}
$$

если $1<j_{i, k}<j_{i+1, k}=m$, и

$$
a_{0, m}\left(\prod_{s=2}^{m-1} a_{r+m-s, m+1-s}^{-1}\right) \bar{w}_{r+1,1, m}=\bar{w}_{r, 1, m}
$$

если $1=j_{i, k}<j_{i+1, k}=m$, где $r \in \mathbb{Z}$ и каждое слово

$$
\bar{w}_{r, j_{i, k}, j_{i+1, k}}=x_{m}^{r} w_{j_{i, k}, j_{i+1, k}, 1} x_{m}^{-\left(r+t_{j_{i, k}}\right)}
$$

записано в алфавите $a_{l, s}$.

Как и в работе [13], можно показать, что $\bar{w}_{r, j_{i, k}, j_{i+1, k}}$ и $\bar{w}_{r+m, j_{i, k}, j_{i+1, k}}$ сопряжены в группе $G_{2}$ с помошью элемента $a_{0, m}$. Следовательно, взяв сумму по $r$, из соотношений $(5.8)-(5.11)$ получим следуюшие соотношения в группе $N_{2} / N_{2}^{\prime}$ :

$$
\sum_{r=1}^{m} \bar{a}_{r, j_{i, k}}=\sum_{r=1}^{m} \bar{a}_{r, j_{i+1, k}}
$$


если $1<j_{i, k}<j_{i+1, k}<m$,

$$
m \bar{a}_{0, m}-\sum_{s=2}^{m-1} \sum_{r=1}^{m} \bar{a}_{r, s}=\sum_{r=1}^{m} \bar{a}_{r, j_{i+1, k}}
$$

если $1=j_{i, k}<j_{i+1, k}<m$,

$$
\sum_{r=1}^{m} \bar{a}_{r, j_{i, k}}=0
$$

если $1<j_{i, k}<j_{i+1, k}=m$, и

$$
m \bar{a}_{0, m}-\sum_{s=2}^{m-1} \sum_{r=1}^{m} \bar{a}_{r, s}=0,
$$

если $1=j_{i, k}<j_{i+1, k}=m$.

Легко видеть, что для любого разложения $\{1, \ldots, m\}=\bigsqcup_{k=1}^{n} J_{k}$ уравнения (5.12)-(5.15) являются линейно независимыми над $\mathbb{Z}$, число этих уравнений равно $m-n$ и элементы $\bar{a}_{0, m}$ и $\sum_{r=1}^{m} \bar{a}_{r, s}, s=2, \ldots, m-1$, порождают группу $\left(N_{1} / N_{1}^{\prime}\right)_{1}$. Следовательно, ранг ядра ограничения $g_{*}$ на $\left(N_{1} / N_{1}^{\prime}\right)_{1}$ не меньше $m-n$. Таким образом,

$$
\operatorname{dim}\left(N_{2} / N_{2}^{\prime} \otimes \mathbb{C}\right)_{1} \leqslant \operatorname{dim}\left(N_{1} / N_{1}^{\prime} \otimes \mathbb{C}\right)_{1}-(m-n)=n-1 .
$$

С другой стороны, согласно лемме 5.8 многочлен Александера $\Delta(t) C$-группы $G$, состоящей из $n$ неприводимых компонент, делится на $(t-1)^{n-1}$.

СлЕДСТВИЕ 5.10. Пусть гурвищевская $C$-группа $G$ состоит из $n$ неприводимых компонент, и пусть $\Delta(t)$ - ее многочлен Александера. Тогда

$$
\Delta(0)=(-1)^{\operatorname{deg} \Delta(t)-(n-1)} .
$$

ДокАЗАТЕЛЬСТво. Многочлен Александера $\Delta(t)$ имеет вид

$$
\Delta(t)=\operatorname{det}\left(h_{\mathbb{C}}-t \mathrm{Id}\right)=(-t)^{\operatorname{deg} \Delta(t)}+\sum_{i=0}^{\operatorname{deg} \Delta(t)-1} c_{i} t^{i},
$$

и согласно теореме 5.9 имеем $\Delta(t)=(t-1)^{n-1} P(t)$, где $P(t) \in \mathbb{Z}[t]$ - некоторый многочлен такой, что $P(1) \neq 0$. Следовательно, многочлен

$$
P(t)=(-1)^{\operatorname{deg} \Delta(t)} \prod_{i} \Phi_{n_{i}}(t)
$$

является (с точностью до знака) произведением некоторых циклотомических многочленов $\Phi_{n_{i}}(t)$ при $n_{i}>1$. Согласно лемме 5.3 имеем $\Phi_{n_{i}}(0)=1$ для всех $n_{i}>1$. Следовательно, $\Delta(0)=(-1)^{n-1}(-1)^{\Delta(t)}=(-1)^{\operatorname{deg} \Delta(t)-(n-1)}$. 
ЛЕмма 5.11. Пусть $j:\{1, \ldots, n\} \rightarrow\{1, \ldots, m\}$ - инбективное отобрахение. Предположим, что $C$-группа $G$ порождена $C$-порождающими әлементами $x_{1}, \ldots, x_{n}$ u $w=\bar{x}_{1} \ldots \bar{x}_{m}$ является квазиположительным словом от $x_{1}, \ldots, x_{n}$ (m. е. $\bar{x}_{k}$ сопряжен некоторому $\left.x_{i_{k}} \in\left\{x_{1}, \ldots, x_{n}\right\}\right)$ таким, что $\bar{x}_{j(i)}=x_{i}$ для $i=1, \ldots, n$. Если әлемент $w$ принадлежит центру группь $G$, то $G$ является гурвицевской $C$-группой степени $m$.

ДокАЗАТЕЛЬСтво. Пусть $G=\left\langle x_{1}, \ldots, x_{n} \mid R\right\rangle-C$-копредставление группы $G$. Положим $J=\{1 \leqslant j \leqslant m \mid j=j(i), i=1, \ldots, n\}$. По предположению имеем $\bar{x}_{j(i)}=x_{i}$ и

$$
\bar{x}_{j}=w_{j}^{-1} x_{i_{j}} w_{j}
$$

для $j \notin J$, где $w_{j}$ - некоторые слова, записанные в алфавите, состоящем из $x_{1}, \ldots$ $\ldots, x_{n}$ и обратных к ним элементов. Заметим, что соотношения (5.16) являются $C$-соотношениями. Следовательно, если мы добавим порождаюшие элементы $\bar{x}_{j}, j \notin J, \mathrm{k}$ множеству порождающих элементов $\left\{x_{1}, \ldots, x_{n}\right\}$ и добавим соотношения (5.16) к множеству $R$, то получим некоторое $C$-копредставление той же самой группы $G$. Для завершения доказательства достаточно перенумеровать полученное в результате добавления множество порождающих.

ПРЕДЛОЖЕНИЕ 5.12. Пусть $G_{1} u G_{2}$ - әурвицевские $C$-группы степени $m_{1}$ и $m_{2}$ соответственно, и пусть $\Delta_{i}(t), i=1,2,-u x$ многочлены Александера. Тогда существует гурвицевская $C$-группа степени $2 m_{1} m_{2}$ с многочленом Александера $\Delta(t)=\Delta_{1}(t) \Delta_{2}(t)$.

ДокАЗАТЕЛЬСтво. Пусть $G_{i}=\left\langle x_{1, i}, \ldots, x_{m_{i}, i} \mid R_{i}\right\rangle$ - гурвищевское $C$-копредставление группы $G_{i}$ степени $m_{i}$.

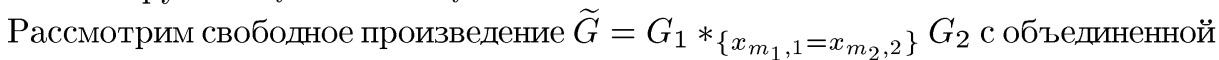
подгруппой. Это произведение является $C$-группой, заданной копредставлением

$$
\widetilde{G}=\left\langle x_{1, i}, \ldots, x_{m_{i}, i}, i=1,2 \mid R_{1} \cup R_{2}, x_{m_{1}, 1}=x_{m_{2}, 2}\right\rangle .
$$

Положим $y_{i}=x_{1, i} \ldots x_{m_{i}, i}, i=1,2$, и обозначим через $N_{i}$ (соответственно, через $\widetilde{N}$ ) ядро канонического гомоморфизма $\nu: G_{i} \rightarrow \mathbb{F}_{1}$ (соответственно, гомоморфизма $\left.\nu: \widetilde{G} \rightarrow \mathbb{F}_{1}\right)$.

Как и в доказательстве теоремы 5.6, применяя метод Рейдемейстера-Шрайера, покажем, что группа $N_{i}$ (соответственно, $\tilde{N}$ ) порождается элементами $a_{k, j, i}=$ $x_{m_{i}, i}^{k} x_{j, i} x_{m_{i}, i}^{-(k+1)}$, где $j=2, \ldots, m_{i}-1, k \in \mathbb{Z}$, вместе с элементами $a_{k, m_{i}, i}=$ $x_{m_{i}, i}^{k} y_{i} x_{m_{i}, i}^{-\left(k+m_{i}\right)}, k \in \mathbb{Z}$ (соответственно, объединением этих элементов, так как $x_{m_{1}, 1}=x_{m_{2}, 2}$ в групше $\widetilde{G}$ ). Множество определяюших соотношений для $N_{i}$ (соответственно, для $\widetilde{N})$ получается из множества $\bar{R}_{i}=\left\{x_{m_{i}, i}^{n} r_{l, i} x_{m_{i}, i}^{-n} \mid r_{l, i} \in R_{i}\right.$, $n \in \mathbb{Z}\}$ (соответственно, из $\bar{R}=\bar{R}_{1} \cup \bar{R}_{2}$ ) после переписывания слов в виде слов из алфавита $\left\{a_{k, j, i}\right\}$ (соответственно, из объединения этих алфавитов). Следовательно, $\widetilde{N}=N_{1} * N_{2}$ является свободным произведением групп $N_{1}$ и $N_{2}$.

Отметим, что из доказательства теоремы 5.6 следует, что элементы $a_{k, j, i}$ и $a_{k+l m_{i}, j, i}$ яВляются сопряженными в группе $N_{i}$ при всех $l \in \mathbb{Z}$. 
Пусть $\tilde{h}_{i}$ - автоморфизм группы $N_{i}$, заданный сопряжением на элемент $x_{m_{i}, i}$. Тогда автоморфизм $\tilde{h}$ групшы $\widetilde{N}$ задается сопряжением на элемент $x_{m_{1}, 1}=x_{m_{2}, 2}$ и этот автоморфизм равен $\tilde{h}_{1} * \tilde{h}_{2}$. Следовательно, многочлен Александера $\tilde{\Delta}(t)$ группы $\widetilde{G}$ равен $\Delta_{1}(t) \Delta_{2}(t)$.

Рассмотрим группу

$$
\begin{gathered}
G=\left\langle x_{1, i}, \ldots, x_{m_{i}, i}, i=1,2\right| R_{1} \cup R_{2}, x_{m_{1}, 1}=x_{m_{2}, 2}, \\
\left.\left[x_{j, i}, y_{\bar{i}}^{m_{i}}\right]=1, j=1, \ldots, m_{i}-1, i=1,2\right\rangle,
\end{gathered}
$$

где $\bar{i}=\{1,2\} \backslash\{i\}$ (напомним, что $x_{1, \bar{i}}, \ldots, x_{m_{\bar{i}}, \bar{i}}$ коммутируют с $\left.y_{\bar{i}}\right)$. Пусть $N-$ ядро канонического гомоморфизма $\nu: G \rightarrow \mathbb{F}_{1}$.

Чтобы получить копредставление группы $N$ из копредставления группы $\widetilde{N}$, описанного выше, нужно добавить соотношения, индуцированные соотношениями

$$
\left[x_{j, i}, y_{\bar{i}}^{m_{i}}\right]=1, \quad j=1, \ldots, m_{i}-1, \quad i=1,2 .
$$

Легко видеть, что эти добавленные соотношения имеют следующий вид:

$$
a_{k, j, i} a_{0, m_{\bar{i}}}^{m_{i}}=a_{0, m_{\bar{i}}}^{m_{i}} a_{k+m_{1} m_{2}, j, i}
$$

так как $a_{k, m_{\bar{i}}}=a_{k+1, m_{\bar{i}}}$ в группе $N_{\bar{i}}$ при всех $k$. Из дополнительных соотношений (5.17) видно, что $a_{k, j, i}$ и $a_{k+m_{1}} m_{2}, j, i$ сопряжены друг другу. Однако эти элементы были уже сопряжены в группе $\widetilde{N}$. Следовательно, $\widetilde{N} / \widetilde{N^{\prime}} \simeq N / N^{\prime}$, и группы $\widetilde{G}$ и $G$ имеют одинаковые многочлены Александера. Для завершения доказательства предложения 5.12 достаточно заметить, что

$$
y_{1}^{m_{2}} y_{2}^{m_{1}}=\left(x_{1,1} \ldots x_{m_{1}, 1}\right)^{m_{2}}\left(x_{1,2} \ldots x_{m_{2}, 2}\right)^{m_{1}}
$$

принадлежит центру группы $G$. Следовательно, согласно лемме 5.11 группа $G$ является гурвищевской $C$-группой степени $2 m_{1} m_{2}$.

Для двух гурвицевских $C$-групп $G_{1}$ и $G_{2}$, заданных гурвицевскими $C$-копредставлениями групп $G_{i}=\left\langle x_{1, i}, \ldots, x_{m_{i}, i} \mid R_{i}\right\rangle, i=1,2$, гурвицевская $C$-группа $G$, построенная в доказательстве предложения 5.12 , называется гурвицевским произведением групп $G_{1}$ и $G_{2}$. Гурвицевское произведение групп $G_{1}$ и $G_{2}$ будем обозначать через $G_{1} \diamond G_{2}$. Конечно, гурвицевское произведение групп $G_{1}$ и $G_{2}$ зависит от гурвицевских $C$-копредставлений груп $G_{1}$ и $G_{2}$, но согласно предложению 5.12 многочлен Александера группы $G_{1} \diamond G_{2}$ не зависит от гурвицевских $C$-копредставлений множителей.

ЛЕмма 5.13. Фундаментальная группа $G_{n, m}=\pi_{1}\left(\mathbb{C}^{2} \backslash C_{n, m}\right)$ дополнения $\kappa$ плоской аффинной алгебраической кривой $C_{m, n}$, заданной уравнением $w^{n}-z^{m}=0$, где п и гурвицевской $C$-группой. 
ЗАмЕчАниЕ 5.14. Лемма 5.13 не следует из упомянутого выше утверждения о фундаментальных группах дополнений к аффинным кривым Гурвица, так как в нем предполагается, что “бесконечно удаленная" прямая находится в общем положении по отношению к кривой Гурвица, а в рассматриваемом случае это не так. Если мы рассмотрим локальную фундаментальную группу $G=\pi_{1}\left(B_{\varepsilon} \backslash C\right)$, где $C$ неприводимая особенность в небольшом шаре $B_{\varepsilon}$, то $G$ всегда имеет естественную структуру неприводимой $C$-группы. Эта группа имеет нетривиальный центр тогда и только тогда, когда особенность $C$ имеет тип $x^{p}=y^{q}$, где $p$ и $q$ взаимно просты (см. [3]).

ДОКАЗАТЕЛЬСТВО ЛЕМмЫ 5.13. Действительно, брэйд-монодромия особенности $w^{n}=z^{m}$ относительно проекции $(z, w) \rightarrow z$ равна

$$
b_{n, m}=\left(\sigma_{1} \ldots \sigma_{n-1}\right)^{m} \in \mathrm{Br}_{n}
$$

где $\sigma_{1}, \ldots, \sigma_{n-1}$ - стандартные порождающие элементы группы кос $\mathrm{Br}_{n}$, т. е. порождающие, связанные следующими соотношениями:

$$
\begin{aligned}
\sigma_{i} \sigma_{i+1} \sigma_{i} & =\sigma_{i+1} \sigma_{i} \sigma_{i+1}, \quad i=1, \ldots, n-2, \\
{\left[\sigma_{i}, \sigma_{j}\right] } & =1, \quad|i-j| \geqslant 2 .
\end{aligned}
$$

Группа $\mathrm{Br}_{n}$ действует на свободной группе $\mathbb{F}_{n}$, порожденной элементами $x_{1}, \ldots$ $\ldots, x_{n}$. Это действие задается формулами $\sigma_{j}\left(x_{i}\right)=x_{i}$, если $j \neq i, i+1$, и $\sigma_{j}\left(x_{j+1}\right)=x_{j}, \sigma_{j}\left(x_{j}\right)=x_{j} x_{j+1} x_{j}^{-1}$. Обозначим через $B_{n, m}$ циклическую подгруппу группы $\mathrm{Br}_{n}$, порожденную элементом $b_{n, m}$. Тогда (см. [11]) группа

$$
G_{n, m}=\left\langle x_{1}, \ldots, x_{n} \mid x_{i}=b\left(x_{i}\right), i=1, \ldots, n, b \in B_{n, m}\right\rangle
$$

является $C$-группой и согласно лемме 5.11 она является гурвицевской $C$-группой, так как $b_{n, m}^{n}=\left(\Delta_{n}^{2}\right)^{m} \in B_{n, m}$, где $\Delta_{n}$ - элемент Гарсайда в групе кос $\mathrm{Br}_{n}$, и, следовательно, элемент $\left(x_{1} \ldots x_{n}\right)^{m}$ принадлежит центру группы $G_{n, m}$ (так как $\Delta_{n}^{2}\left(x_{i}\right)=\left(x_{1} \ldots x_{n}\right) x_{i}\left(x_{1} \ldots x_{n}\right)^{-1}$ и $\left.\Delta_{n}^{2}\left(x_{1} \ldots x_{n}\right)=x_{1} \ldots x_{n}\right)$.

ПРЕДЛОЖЕНИЕ 5.15 [14]. Если челье числа $m$ и $n$ являются взаимно простыми, то многочлен Александера группьи $G_{n, m}$ имеет вид

$$
\Delta_{n, m}(t)=\frac{(t-1)\left(t^{n m}-1\right)}{\left(t^{n}-1\right)\left(t^{m}-1\right)}
$$

ПРЕДЛОЖЕНИЕ 5.16. Многочлен Александера группь $G_{2,2 m}$ имеет вид

$$
\Delta(t)=(1-t) \sum_{i=0}^{m-1} t^{2 i}
$$


ДокАЗАТЕЛЬСТво. Из доказательства леммы 5.13 следует, что

$$
G_{2,2 m}=\left\langle x_{1}, x_{2} \mid\left[x_{1},\left(x_{1} x_{2}\right)^{m}\right]=\left[x_{2},\left(x_{1} x_{2}\right)^{m}\right]=1\right\rangle .
$$

Покажем, что соотношения

$$
\left[x_{2},\left(x_{1} x_{2}\right)^{m}\right]=1, \quad i=1,2,
$$

эквивалентны одному соотношению $\left(x_{1} x_{2}\right)^{m}=\left(x_{2} x_{1}\right)^{m}$. Действительно, соотношения (5.18) влекут

$$
\left(x_{1} x_{2}\right)^{m}=x_{1}^{-1}\left(x_{1} x_{2}\right)^{m} x_{1}=\left(x_{2} x_{1}\right)^{m},
$$

а соотношение $\left(x_{1} x_{2}\right)^{m}=\left(x_{2} x_{1}\right)^{m}$ влечет

$$
x_{2}\left(x_{1} x_{2}\right)^{m}=\left(x_{2} x_{1}\right)^{m} x_{2}=\left(x_{1} x_{2}\right)^{m} x_{2}
$$

и

$$
x_{1}\left(x_{1} x_{2}\right)^{m}=x_{1}\left(x_{2} x_{1}\right)^{m}=\left(x_{1} x_{2}\right)^{m} x_{1} .
$$

Следовательно,

$$
G_{2,2 m}=\left\langle x_{1}, x_{2} \mid\left(x_{1} x_{2}\right)^{m}\left(x_{2} x_{1}\right)^{-m}=1\right\rangle .
$$

Пусть $r=\left(x_{1} x_{2}\right)^{m}\left(x_{2} x_{1}\right)^{-m}$. Применяя свободное дифференциальное исчисление Фокса [4], получим

$$
\begin{aligned}
& \nu_{*}\left(\frac{\partial r}{\partial x_{1}}\right)=1+t^{2}+\cdots+t^{2(m-1)}-t^{2 m-1}-t^{2 m-3}-\cdots-t^{1}, \\
& \nu_{*}\left(\frac{\partial r}{\partial x_{2}}\right)=t+\cdots+t^{2 m-1}-t^{2(m-1)}-t^{2(m-2)}-\cdots-t^{2}-1 .
\end{aligned}
$$

Следовательно,

$$
\Delta(t)=(1-t) \sum_{i=0}^{m-1} t^{2 i}
$$

Рассмотрим группу

$$
\begin{aligned}
G(2)= & \left\langle x_{1}, \ldots, x_{4}\right| x_{2}^{2} x_{1} x_{2}^{-2}=x_{4}, x_{3}=x_{2}, x_{4}^{2} x_{2} x_{4}^{-2}=x_{2}, \\
& {\left.\left[x_{i}, x_{1} \ldots x_{4}\right]=1 \text { для } i=1, \ldots, 4\right\rangle . }
\end{aligned}
$$

ПРЕДЛОЖЕНИЕ 5.17. Многочлен Александера $\Delta(t)$ группь $G(2)$ равен $t^{2}-1$. 
ДоКАЗАТЕЛЬСТво. Обозначим через $N(2)$ ядро канонического $C$-гомоморфизма $\nu: G(2) \rightarrow \mathbb{F}_{1}$ и положим $m=4$ и $y=x_{1} \ldots x_{4}$. В обозначениях, используемых в доказательстве теоремы 5.6, из соотношений $\left[x_{i}, y\right]=1$ для $i=1, \ldots, 4$ следует, что группа $N(2)$ порождается элементами $a_{k, j}=x_{4}^{k} x_{j} x_{4}^{-(k+1)}, k=1, \ldots, 4$, $j=2,3$, вместе с элементом $a_{0,4}=y x_{4}^{-4}$. В нашем случае соотношения $(5.5)$ и (5.6) имеют вид

$$
a_{k, 4}=a_{0,4}, \quad a_{k+4, j}=a_{0,4}^{-1} a_{k, j} a_{0,4}
$$

для всех $k$.

Из соотношения $x_{3}=x_{2}$ следуют соотношения

$$
a_{k, 3}=a_{k, 2}
$$

для всех $k$, а из соотношения $x_{4}^{2} x_{2}=x_{2} x_{4}^{2}-$ соотношения

$$
a_{k+2,2}=a_{k, 2}
$$

для всех $k$.

Из соотношения $x_{2}^{2} x_{1}=x_{4} x_{2}^{2}$, записанного как $x_{2}^{2} y=x_{4} x_{2}^{4} x_{4}\left(\right.$ так как $\left.x_{2}=x_{3}\right)$, имеем соотношения

$$
a_{k, 2} a_{k+1,2} a_{k+2,4}=a_{k+1,2} a_{k+2,2} a_{k+3,2} a_{k+4,2}
$$

для всех $k$.

Из (5.20)-(5.23) следует, что группа $N(2)$ порождается элементами $a_{1,2}, a_{2,2}$ и $a_{0,4}$, которые удовлетворяют соотношениям

$$
a_{0,4}=\left(a_{1,2} a_{2,2}\right)^{-1}\left(a_{2,2} a_{1,2}\right)^{2}=\left(a_{2,2} a_{1,2}\right)^{-1}\left(a_{1,2} a_{2,2}\right)^{2}
$$

И

$$
\left[a_{1,2}, a_{0,4}\right]=\left[a_{2,2}, a_{0,4}\right]=1 .
$$

Следовательно, группа $N(2) / N(2)^{\prime}$ является свободной абелевой группой, порожденной образами $\bar{a}_{1,2}$ и $\bar{a}_{2,2}$ элементов $a_{1,2}$ и $a_{2,2}$.

Как и в доказательстве теоремы 5.6 , действие $\tilde{h}$ на $N(2)$ задается формулами $\tilde{h}\left(a_{1,2}\right)=a_{2,2}, \tilde{h}\left(a_{2,2}\right)=a_{3,2}=a_{1,2}$. Индуцированное действие $h$ на $N(2) / N(2)^{\prime}$ задается формулами $h\left(\bar{a}_{1,2}\right)=\bar{a}_{2,2}, h\left(\bar{a}_{2,2}\right)=\bar{a}_{1,2}$, и характеристический многочлен этого действия равен $(t-1)(t+1)$.

СЛЕДСТВИЕ 5.18. Для любого $k \in \mathbb{N}$ существует гурвицевская $C$-группа $G$, состоящая из двух неприводимых компонент, имеющая многочлен Александера $\Delta(t)=(t-1) P(t)$ такой, что $|P(1)|=k$.

ДоКАЗАТЕЛЬСТВО. Если $k>2$, то согласно предложению 5.16 имеем $P(1)=-k$, где $P(t)=-\sum_{i=0}^{k-1} t^{2 i}$ является множителем многочлена Александера $\Delta(t)=(1-t) \sum_{i=0}^{k-1} t^{2 i}$ группы $G_{2,2 k}$. Если $k=2$, то согласно предложению 5.17 группа $G(2)$ обладает требуемым свойством, так как ее многочлен Александера $\Delta(t)=(t-1)(t+1)$. В случае $k=1$ можно взять абелеву гурвицевскую $C$-группу $G=\mathbb{Z}^{2}$. 
ПРЕДЛОЖЕНИЕ 5.19. Для любого $k \in \mathbb{N}$ существуют:

(i) неприводимая гурвичевская $C$-группа, многочлен Александера $\Delta(t)$ которой имеет степень $\operatorname{deg} \Delta(t)=2 k$;

(ii) гурвичевская $C$-группа, состоящая из двух неприводимых компонент, многочлен Александера которой есть $\Delta(t)=(t-1) P(t)$, где многочлен $P(t)$ имеет степень $\operatorname{deg} P(t)=k$.

ДоКАЗАТЕльСтво. Согласно предложениям 5.12 и 5.15 многочлен Александеpa $\Delta(t)$ гурвищевского произведения $G_{2}^{\diamond k}$, равен $\left(t^{2}-t+1\right)^{k}$.

Чтобы доказать утверждение (ii), достаточно взять гурвищевские $C$-группы $G(2) \diamond G_{2,3}^{\diamond n}$, если $k=2 n+1$, т.е. является нечетным числом, и $\mathbb{Z}^{2} \diamond G_{2,3}^{\diamond n}$, если $k=2 n$, т. е. четно.

ВоПРОС 5.20. Пусть $P(t) \in \mathbb{Z}[t]-$ многочлен, все корни которого являются корнями из единицы, $t=1$ является корнем многочлена $P(t)$ кратносmи $k$, и пусть $P(0)=(-1)^{\operatorname{deg} P(t)-k}$. Предположим также, что $P(1)=1$, если $k=0$. Существует ли гурвицевская $C$-группа $G$, имеющая многочлен Александера $\Delta(t)=P(t)$ ?

\section{§6. Первое число Бетти циклических накрытий плоскости}

Рассмотрим циклическое накрытие $f=f_{\infty}: X_{\infty} \rightarrow X^{\prime}=\mathbb{C}^{2} \backslash H$, соответствующее каноническому эпиморфизму $\nu: \pi_{1}\left(\mathbb{C}^{2} \backslash H\right) \rightarrow \mathbb{F}_{1}$. Пусть $h \in \operatorname{Deck}\left(X_{\infty} / X^{\prime}\right) \simeq$ $\mathbb{F}_{1}$ - накрывающее преобразование, соответствующее $C$-порождающему элементу $x \in \mathbb{F}_{1}$. Будем называть $h$ монодромией кривой Гурвица $H$. Пространство $X^{\prime}$ будем рассматривать как факторпространство $X^{\prime}=X_{\infty} / \mathbb{F}_{1}$. В этой ситуации Милнор [17] рассмотрел точную последовательность цепных комплексов

$$
0 \rightarrow C .\left(X_{\infty}\right) \stackrel{h-\mathrm{id}}{\longrightarrow} C .\left(X_{\infty}\right) \stackrel{\varphi_{*}}{\longrightarrow} C .\left(X^{\prime}\right) \rightarrow 0
$$

которая приводит к точной последовательности групп гомологий

$$
\cdots \rightarrow H_{1}\left(X_{\infty}\right) \stackrel{h-\mathrm{id}}{\longrightarrow} H_{1}\left(X_{\infty}\right) \stackrel{f_{*}}{\longrightarrow} H_{1}\left(X^{\prime}\right) \rightarrow H_{0}\left(X_{\infty}\right) \rightarrow 0
$$

(будем писать $h$ вместо $h_{*}$, когда это не приводит к недоразумению).

Если $G_{n} \subset \mathbb{F}_{1}$ - бесконечная циклическая группа, порожденная элементом $h^{n}$, то $X_{n}^{\prime}=X_{\infty} / G_{n}$ и $X^{\prime}=X_{n}^{\prime} / \mu_{n}$, где $\mu_{n}=\mathbb{F}_{1} / G_{n}$ - циклическая группа порядка $n$. Обозначим через $h_{n}$ автоморфизм пространства $X_{n}^{\prime}$, индуцированный монодромией $h$. Тогда $h_{n}$ является порождаюшим элементом группы накрывающих преобразований $\operatorname{Deck}\left(X_{n}^{\prime} / X^{\prime}\right)=\mu_{n}$, действующей на $X_{n}^{\prime}$. Применим последовательность

$$
\cdots \rightarrow H_{1}\left(X_{\infty}\right) \stackrel{h^{n}-\mathrm{id}}{\longrightarrow} H_{1}\left(X_{\infty}\right) \stackrel{g_{n, *}}{\longrightarrow} H_{1}\left(X_{n}^{\prime}\right) \rightarrow H_{0}\left(X_{\infty}\right) \rightarrow 0,
$$

построенную аналогично последовательности (6.1), к бесконечному циклическому накрытию $g_{n}=g_{\infty, n}: X_{\infty} \rightarrow X_{n}^{\prime}$ для исследования группы $H_{1}\left(X_{n}^{\prime}\right)$. 
Обозначим через $H_{1}\left(X_{\infty}, \mathbb{C}\right)_{n}$ подпространство векторного пространства $H_{1}\left(X_{\infty}, \mathbb{C}\right)$, порожденное собственными векторами автоморфизма $h_{*}$, которые имеют собственные значения $\lambda$, являющиеся корнями степени $n$ из единицы, и через $H_{1}\left(X_{\infty}, \mathbb{C}\right)_{n, \neq 1}$ - векторное подпространство, порожденное собственными векторами, которые имеют собственные значения $\lambda \neq 1$, являющиеся корнями степени $n$ из единицы. Согласно теореме 5.1 имеем $\operatorname{dim} H_{1}\left(X_{\infty}, \mathbb{C}\right)_{n}=r_{n}$ и $\operatorname{dim} H_{1}\left(X_{\infty}, \mathbb{C}\right)_{n, \neq 1}=r_{n, \neq 1}$, где $r_{n}$ (соответственно, $\left.r_{n, \neq 1}\right)-$ число корней многочлена Александера $\Delta(t)$ кривой Гурвища $\bar{H}$, являюшихся (соответственно, не равными 1) корнями степени $n$ из единицы. Отметим, что согласно лемме 5.7 и теореме 5.9 выполнено

$$
r_{n}-r_{n, \neq 1}=r_{1}=\#\{\text { неприводимые компоненты кривой } \bar{H}\}-1 \text {. }
$$

ПРЕДЛОЖЕНИЕ 6.1. Имеем:

(i) $\operatorname{dim} H_{1}\left(X_{n}^{\prime}, \mathbb{C}\right)=r_{n}+1$;

(ii) $\operatorname{dim} H_{1}\left(X_{n}^{\prime}, \mathbb{C}\right)_{1}=r_{1}+1=\#\{$ неприводимые компоненты кривой $\bar{H}\}$.

ДОКАЗАТЕЛЬСТВо следует из точной последовательности (6.2).

Пусть $\bar{H}$ - кривая Гурвища, состояшая из $k$ неприводимых компонент $\bar{H}_{1}, \ldots$ $\ldots, \bar{H}_{k}$. Выберем прямую $L \subset \mathbb{C}^{2}$, принадлежашую пучку прямых (относительно которого кривая $\bar{H}$ определена) и трансверсально пересекающую кривую $H$. Обозначим через $\gamma_{i}$ окружность малого радиуса, лежашую в $L$, с центром в одной из точек пересечения $H_{i} \cap L$. Легко видеть, что циклы $\gamma_{1}, \ldots, \gamma_{k}$, соответствующие выбранным окружностям, образуют базис группы $H_{1}\left(\mathbb{C}^{2} \backslash H, \mathbb{Z}\right)$ и не зависят от выбора прямой $L$. Пусть $\bar{\gamma}_{i}-$ цикл в $H_{1}\left(X_{n}^{\prime}, \mathbb{Z}\right)$, соответствующий простому пути $f_{n}^{-1}\left(\gamma_{i}\right), i=1, \ldots, k$.

ЛЕмма 6.2. Цикль $\bar{\gamma}_{i}, \quad i=1, \ldots, k$, являются линейно независимыми в $H_{1}\left(X_{n}^{\prime}, \mathbb{Z}\right)$ и образуют базис в $H_{1}\left(X_{n}^{\prime}\right)_{1}$.

ДокАЗАТЕЛЬСТво. Очевидно, все циклы $\bar{\gamma}_{i}$ инвариантны при действии $h_{n}$. Поэтому доказательство следует из предложения 6.1 , (ii) и из замечания о том, что при гомоморфизме $\left(f_{n}\right)_{*}: H_{1}\left(X_{n}^{\prime}, \mathbb{Z}\right) \rightarrow H_{1}\left(\mathbb{C}^{2} \backslash H, \mathbb{Z}\right)$ мы имеем $\left(f_{n}\right)_{*}\left(\bar{\gamma}_{i}\right)=n \gamma_{i}$.

В обозначениях из доказательства теоремы 4.1 накрытие $f_{n}$ может быть продолжено до отображения $\tilde{f}_{n}: \widetilde{X}_{n} \rightarrow \mathbb{C P}^{2}$, разветвленного вдоль $\bar{H}$ и, возможно, вдоль $\bar{L}_{\infty}$, где $\widetilde{X}_{n}$ является замкнутым четырехмерным многообразием, локально изоморфным над каждой особой точкой кривой $\bar{H}$ комплексно-аналитической особенности, заданной уравнением $w_{1}^{n}=\widetilde{F}_{1}\left(u_{1}, v_{1}\right)$, где

$$
\widetilde{F}_{1}\left(u_{1}, v_{1}\right)=\prod_{j}\left(v_{1}-v_{1, j}\left(u_{1}\right)\right)
$$

и произведение взято по всем ветвям кривой $\bar{H}$, замыкание которых содержит эту особую точку. Кроме того, $\widetilde{X}_{n}$ над каждой точкой пересечения кривой $\bar{H}$ и прямой $L_{\infty}$ локально изоморфно особенности, заданной уравнением $w_{2}^{n}=\left(v_{2}-e^{\frac{2 \pi i}{m}}\right) u^{d}$, где $d$ - наименьшее неотрищательное целое число, для которого число $m+d$ делится на $n$. Многообразие $\widetilde{X}_{n}$, если $\tilde{f}_{n}^{-1}\left(L_{\infty}\right) \subset \operatorname{Sing} \widetilde{X}_{n}$, может быть нормализовано 
(как и в алгебраическом случае), и мы получаем накрытие $\tilde{f}_{n, \text { norm }}: \widetilde{X}_{n, \text { norm }} \rightarrow$ $\mathbb{C P}^{2}$, в котором $\widetilde{X}_{n, \text { nоrm }}$ является особым аналитическим многообразием в его особых точках, число которых конечно. Мы можем разрешить эти особые точки и получить гладкое многообразие $\bar{X}_{n}$. Пусть $\sigma: \bar{X}_{n} \rightarrow \widetilde{X}_{n, \text { norm }}-$ разрешение особых точек, $E=\sigma^{-1}\left(\operatorname{Sing} \widetilde{X}_{n, \text { norm }}\right)$ и $\bar{f}_{n}=\tilde{f}_{n, \text { norm }} \circ \sigma$. Пусть $R_{i}=\tilde{f}_{n, \text { norm }}^{-1}\left(\bar{H}_{i}\right)$, $i=1, \ldots, k$, и $R_{\infty}=\tilde{f}_{n, \text { norm }}^{-1}\left(L_{\infty}\right)$. Заметим, что ограничение отображения

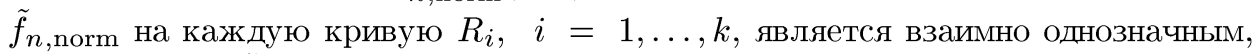

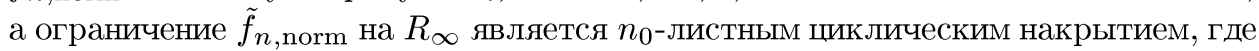
$n_{0}=\operatorname{HОД}(n, d)$ и индекс ветвления $n_{\infty}$ отображения $\tilde{f}_{n, \text { norm }}$ вдоль $R_{\infty}$ равен $\frac{n}{n_{0}}$. Как и в алгебраическом случае, легко показать, что кривая $R_{\infty}$ является неприводимой. Обозначим через $\bar{R}_{i}=\sigma^{-1}\left(R_{i}\right), i=1, \ldots, k, \infty$, собственный прообраз кривой $R_{i}$.

Имеем вложения $i_{1}: X_{n}^{\prime} \hookrightarrow X_{n}=\bar{X}_{n} \backslash E$ и $i_{2}: X_{n} \hookrightarrow \bar{X}_{n}$.

ЛЕмма 6.3. Индуиированный гомоморфизм $i_{1 *}: H_{1}\left(X_{n}^{\prime}\right) \rightarrow H_{1}\left(X_{n}\right)$ является эпиморфизмом, ядро которого $\operatorname{ker} i_{1 *}=H_{1}\left(X_{n}^{\prime}\right)_{1}$.

ДокАЗАТЕЛЬСТво. Имеем

$$
X_{n}^{\prime}=X_{n} \backslash\left(\bigcup_{i=1}^{k} \bar{R}_{i}\right) \cup \bar{R}_{\infty}
$$

и каждая кривая $\bar{R}_{i}, i=1, \ldots, k, \infty$, является подмногообразием коразмерности два в $X_{n}$. Поэтому каждый одномерный цикл $\gamma \subset X_{n}$ может быть сдвинут в дополнение к $\left(\bigcup_{i=1}^{k} \bar{R}_{i}\right) \cup \bar{R}_{\infty}$. Следовательно, $i_{1, *}$ является эпиморфизмом.

Пусть комплексная прямая $L \subset \mathbb{C P}^{2}$ пересекает прямую $L_{\infty}$ в точке $q \in L_{\infty} \backslash \bar{H}$, и пусть $\gamma_{\infty}-$ небольшая простая петля вокруг $L_{\infty}$, лежашая в $L$. Тогда $f_{n}^{-1}\left(\gamma_{\infty}\right)$ распадается в несвязное объединение $n_{0}$ простых петель $\bar{\gamma}_{\infty, i}, i=1, \ldots, n_{0}$. Поскольку кривая $R_{\infty}$ неприводима, каждые две петли $\bar{\gamma}_{\infty, i}$ и $\bar{\gamma}_{\infty, j}$ принадлежат одному и тому же классу гомологий в $H_{1}\left(X_{n}^{\prime}\right)$ (обозначим его $\bar{\gamma}_{\infty}$ ). Следовательно, $n_{0} \bar{\gamma}_{\infty} \in H_{1}\left(X_{n}^{\prime}\right)_{1}$. Теперь лемма следует из замечания о том, что $\bar{\gamma}_{1}, \ldots, \bar{\gamma}_{k}, \bar{\gamma}_{\infty}$ порождают ядро ker $i_{1 *}$ и $\bar{\gamma}_{1}, \ldots, \bar{\gamma}_{k}$ порождают $H_{1}\left(X_{n}^{\prime}\right)_{1}$.

Лемма 6.4. Гомоморфизм $i_{2 *}: H_{1}\left(X_{n}, \mathbb{C}\right) \rightarrow H_{1}\left(\bar{X}_{n}, \mathbb{C}\right)$ является изоморфизмом.

ДоказАтельство. Имеем $X_{n}=\bar{X}_{n} \backslash E$. Обозначим через $T \subset \bar{X}_{n}$ замкнутую регулярную окрестность дивизора $E$, и пусть $\partial T$ - ее граница, $T^{\prime}=T \backslash E$ и $T^{0}=T \backslash \partial T$. Известно (см., например, доказательство предложения 3.4 в [5]), что гомоморфизм $i_{*}: H_{1}(\partial T, \mathbb{C}) \rightarrow H_{1}(T, \mathbb{C})$, индуцированный вложением $i: \partial T \hookrightarrow T$, является изоморфизмом и, кроме того, существует деформационная ретракция $T^{\prime} \searrow \partial T$. Следовательно, существует деформационная ретракция $X_{n} \searrow X_{n}^{0}$, где $X_{n}^{0}=X_{n} \backslash T^{0}$. Поэтому лемма следует из точной последовательности Майера-Виеториса

$$
H_{2}\left(\bar{X}_{n}\right) \rightarrow H_{1}(\partial T) \rightarrow H_{1}(T) \oplus H_{1}\left(X_{n}^{0}\right) \rightarrow H_{1}\left(\bar{X}_{n}\right) \rightarrow 0 .
$$

Доказательство теоремы 0.4 следует из лемм 6.3, 6.4 и предложения 6.1. 
ПРЕДЛОЖЕНИЕ 6.5. Для любого $k \in \mathbb{N}$ существуют:

(i) неприводимая кривая Гурвица $\bar{H}_{k}$ такая, что разрешение $\bar{X}_{k, 6}$ особенностей ииклического накрытия плоскости $\mathbb{C P}^{2}$ степени шесть, разветвленного вдоль $\bar{H}_{k}$, имеет первое число Бетти $b_{1}\left(\bar{X}_{k, 6}\right)=2 k$;

(ii) кривая Гурвица $\bar{H}_{k}$, состоящая из двух неприводимых компонент, такая, что первое число Бетти $b_{1}\left(\bar{X}_{k, 6}\right)$ разрешения $\bar{X}_{k, 6}$ особенностей ииклического накрытия степени шесть, разветвленного вдоль $\bar{H}$, равно $k$.

ДоКАЗАТЕЛЬСТво. При доказательстве предложения 5.19 было показано, что многочлен Александера $\Delta(t)$ гурвищевского произведения $G_{2,3}(k)=G_{2,3}^{\diamond k}$ равен $\left(t^{2}-t+1\right)^{k}$ и что многочлены Александера $\Delta(t)$ гурвицевских произведений $G_{2,3}(2, n)=G(2) \diamond G_{2,3}^{\diamond n}$ и $G_{2,3}(\mathrm{ab}, n)=\mathbb{Z}^{2} \diamond G_{2,3}^{\diamond n}$ соответственно равны $(t-1) \times$ $(t+1)\left(t^{2}-t+1\right)^{n}$ и $(1-t)\left(t^{2}-t+1\right)^{n}$.

Группы $G_{2,3}(k), G_{2,3}(2, n)$ и $G_{2,3}(\mathrm{ab}, n)$ являются гурвицевскими $C$-группами. Кроме того, мы можем предполагать, что степени этих гурвицевских $C$-групп делятся на шесть (можно взять $y^{6}$, где $y$ - произведение $C$-порождающих в гурвицевском $C$-копредставлении группы, и применить лемму 5.11). Следовательно, согласно $[11$, теорема 6.2$]$ каждая из этих групп может быть реализована как фундаментальная группа $\pi_{1}\left(\mathbb{C}^{2} \backslash H\right)$ для некоторой кривой Гурвища степени, делящейся на шесть. Кривая $\bar{H}$ является неприводимой в случае групा $G_{2,3}(k)$ и состоит из двух неприводимых компонент в остальных двух случаях. Поэтому из теоремы 0.4 вытекает предложение 6.5 .

ПРЕДЛОЖЕнИЕ 6.6. Для любого $k \in \mathbb{N}$ существует кривая Гурвица $\bar{H}_{k}$, состоящая из $k+1$ неприводимых компонент, имеющая особые точки типа $w^{2^{3 k-1}}-z^{2^{3 k-1}}=0$ иявляюшаяся кривой ветвления двулистного ииклического накрытия $\bar{f}_{2}: \bar{X}_{k, 2} \rightarrow \mathbb{C P}^{2}$ c $b_{1}\left(\bar{X}_{k, 2}\right)=k$.

В частности, кривая Гурвица $\bar{H}_{1}$ имеет $\operatorname{deg} \bar{H}_{1}=2^{10}$, число ее особых точек равно $2^{16}$, и все эти точки являются особыми точками типа $w^{4}-z^{4}=0$.

ДокАЗАТЕЛЬСТво. Гурвицевское произведение $G(2, k)=G(2)^{\diamond k}$ является гурвищевской $C$-группой степени $m=2^{3 k-1}$. Согласно предложениям 5.12 и 5.17 ее многочлен Александера - это

$$
\Delta_{k}(t)=(t-1)^{k}(t+1)^{k} .
$$

Согласно [11, теорема 6.2] каждая группа $G(2, k)$ может быть реализована как фундаментальная группа $\pi_{1}\left(\mathbb{C}^{2} \backslash H_{k}\right)$ для некоторой кривой Гурвища $\bar{H}_{k}$ четной степени и с особыми точками типа $w^{m}-z^{m}=0$. Поскольку кратность корня $t=1$ многочлена Александера $\Delta_{k}(t)$ равна $k$, из леммы 5.7 и теоремы 5.9 следует, что кривая $\bar{H}_{k}$ состоит из $k+1$ неприводимых компонент.

Согласно теореме 0.4 первое число Бетти $b_{1}\left(\bar{X}_{k, 2}\right)=k$, так как кратность корня $t=-1$ многочлена Александера $\Delta_{k}(t)$ равна $k$.

Чтобы доказать существование кривой Гурвица $\bar{H}_{1}$ с требуемыми свойствами, достаточно найти некоторое целое число $m$ и некоторое брэйд-монодромное разложение на множители (см. [12])

$$
\Delta_{m}^{2}=b_{1} \cdot \ldots \cdot b_{n}
$$


кривой Гурвища $\bar{H}$ такое, что группа, заданная копредставлением

$$
\left.\left\langle x_{m}, \ldots, x_{m}\right| x_{i}=b_{j}\left(x_{i}\right) \text { для } i=1, \ldots, m, j=1, \ldots, n\right\rangle,
$$

является $C$-изоморфной группе $G(2)$.

Чтобы найти такое копредставление, напомним кратко доказательство теоремы 6.2 из [11] и применим его для вычисления инвариантов кривой Гурвища $\bar{H}_{1}$, для которой $\pi_{1}\left(\mathbb{C} \backslash H_{1}\right) \simeq G(2)$.

Группа $G(2)$ задана копредставлением (5.19). Рассмотрим группу

$$
\left.G_{4,4} \simeq\left\langle x_{1}, \ldots, x_{4}\right| x_{i}=\Delta_{4}^{2}\left(x_{i}\right) \text { для } i=1, \ldots, 4\right\rangle,
$$

где $\Delta_{4}$ - элемент Гарсайда в группе кос $\mathrm{Br}_{4}$. Kоса $\Delta_{4}^{2}$ является брэйд-монодромией особенности, заданной уравнением $w^{4}-z^{4}=0$, и $s_{0}=\Delta_{4}^{2}$ (разложение на множители с единственным множителем) является брэйд-монодромным разложением на множители четырех прямых в $\mathbb{C P}^{2}$, пересекающихся в одной точке.

Чтобы получить копредставление (5.19), необходимо добавить соотношения

$$
x_{2}^{2} x_{1} x_{2}^{-2}=x_{4}, \quad x_{4}^{2} x_{2} x_{4}^{-2}=x_{2}, \quad x_{3}=x_{2}
$$

в копредставление (6.3). Для этого в обозначениях и терминологии из [11] сначала необходимо осуществить несколько раз удвоение (см. [11, теорема 3.2]) брэйд-монодромного разложения на множители $s_{0}=\Delta_{4}^{2}$ для того, чтобы иметь возможность раздвинуть порождающие элементы $x_{1}, \ldots, x_{4}$ и заменить каждое соотношение из (6.4) на соотношения $x_{i}=x_{i+4}$ и соотношения, имеюшие вид $x_{i}=b\left(x_{i}\right)$, где $b-$ некоторая коса, сопряженная стандартному порождающему элементу группы кос. Легко видеть, что в нашем случае достаточно осуществить два раза операцию удвоения, и мы получим брэйд-монодромное разложение на множители $s_{1}=d^{2}\left(s_{0}\right)$ элемента $\Delta_{16}^{2}$ (удвоение $d^{2}\left(s_{0}\right)$ определено в [11] формулой $\left.(25)\right)$, каждый множитель которого либо сопряжен элементу $\Delta_{4}^{2}$, либо сопряжен стандартному порождающему элементу группы $\mathrm{Br}_{16}$, и группа

$$
\left.\left\langle x_{1}, \ldots, x_{16}\right| x_{i}=b\left(x_{i}\right), i=1, \ldots, 16, \text { и } b \text { - множители в } s_{1}\right\rangle
$$

$C$-изоморфна групп $G_{4,4}$. Число множителей в $s_{1}$, сопряженных элементу $\Delta_{4}^{2}$, равно $4^{2}$.

Затем, чтобы добавить соотношения (6.4) к копредставлению (6.3), достаточно три раза применить лемму 3.4 из [11] и получить брэйд-монодромное разложение на множители $s_{2}$ элемента $\Delta_{2}^{2}$, каждьй множитель которого сопряжен либо элементу $\Delta_{4}^{2}$, либо стандартному порождающему элементу групшы $\mathrm{Br}_{2} 10$. Число множителей разложения $s_{2}$, сопряженных элементу $\Delta_{4}^{2}$, равно $4^{8}$. Разложение $s_{2}$ является брэйд-монодромным разложением на множители кривой Гурвица $\bar{H}_{1}, \operatorname{deg} \bar{H}_{1}=2^{10}$, кривая $\bar{H}_{1}$ имеет $4^{8}$ особых точек вида $w^{4}-z^{4}=0$, и $\pi_{1}\left(\mathbb{C}^{2} \backslash H\right) \cong G(2)$ по построению разложения $s_{2}$. 


\section{Список литературы}

1. Auroux D. Symplectic 4-manifolds as branched coverings of $\mathbb{P}^{2} / /$ Invent. Math. 2000. V. 139. P. 551-602.

2. Auroux D., Katzarkov L. Branched coverings of $\mathbb{P}^{2}$ and invariants of symplectic 4-manifolds // Invent. Math. 2000. V. 142. P. 631-673.

3. Burde G., Zieschang H. Knots. Berlin-N.Y.: Walter de Gruyter, 1985.

4. Crowell R., Fox R. Knot Theory. Boston: Ginn, 1963.

5. Dimca A. Singularities and Topology of Hypersurfaces. Berlin-N.Y: Springer-Verlag, 1992.

6. Esnault E. Fibre de Milnor d'un cone sur une courbe plane singuliére // Invent. Math. 1982. V. 68. P. 477-496.

7. Kohno T. An algebraic computation of the Alexander polynomial of a plane algebraic curve // Proc. Japan Acad. Ser. A. Math. Sci. 1983. V. 59. P. 94-97.

8. Куликов B.C., Куликов Вик. C. О монодромии и смешанной структуре Ходжа в когомологиях бесконечного циклического накрытия дополнения к плоской кривой // Изв. РАН. Сер. матем. 1995. Т. 59. № 2. С. 143-162.

9. Куликов Вик. С. Многочлены Александера плоских алгебраических кривых // Изв. РАН. Сер. матем. 1993. Т. 57. № 1. С. 76-101.

10. Куликов Вик. C. Геометрическая реализация $C$-групп // Изв. РАН. Сер. матем. 1994. T. 58. № 4. C. 194-203.

11. Куликов Вик. C. Формула разложения на множители полного поворота с удвоенным числом нитей // Изв. РАН. Сер. матем. 2004. Т. 68. № 1. С. 123-158.

12. Куликов Вик. С., Харламов В. М. О брэйд-монодромных разложениях на множители // Изв. РАН. Сер. матем. 2003. Т. 67. № 3. С. 79-118.

13. Куликова О.В. О фундаментальных группах дополнений к кривым Гурвица // Изв. РАН. Сер. матем. 2005. Т. 69. № 1. C. 125-132.

14. Lê Dũng Tráng. Sur les noeuds algébriques // Compositio Math. 1972. V. 25. P. 281-321.

15. Libgober $A$. Alexander polynomials of plane algebraic curves and cyclic multiple planes // Duke Math. J. 1982. V. 49. P. 833-851.

16. McDuff D., Salamon D. Introduction to Symplectic Topology (second edition). Oxford Math. Monographs. Oxford: Clarendon Press, 1998.

17. Milnor J. Infinite cyclic coverings // Conf. on the Topology of manifolds. Boston: Weber \& Schmidt, 1968. P. 115-133.

18. Moishezon B. The arithmetic of braids and a statement of Chisini // Contemporary Math. 1994. V. 164. P. 151-175.

19. Rolfsen D. Knots and Links // Math. Lect. Series 7. Houston: Publish or Perish, 1990.

20. Randell $R$. Milnor fibres and Alexander polynomials of plane curves // Proc. Symp. Pure Math. 40. Part 2. (Arcata Singularities Conference). AMS, 1983. P. 415-420.

21. Stein K. Analytische Zerlegungen komplexer Räume // Math. Ann. 1956. V. 132. P. 63-93.

22. Zariski O. Algebraic surfaces. N.Y.: Springer-Verlag, 1971.

Университет г. Кайзерслаутерн

Математический институт им. ВА. Стеклова РАН

Поступило в редакцию

E-mail: greuel@mathematik.uni-kl.de, kulikov@mi.ras.ru 